\title{
ALMOST INNER DERIVATIONS OF LIE ALGEBRAS
}

\author{
DIETRICH BURDE, KAREL DEKIMPE, AND BERT VERBEKE
}

\begin{abstract}
We study almost inner derivations of Lie algebras, which were introduced by Gordon and Wilson in their work on isospectral deformations of compact solvmanifolds. We compute all almost inner derivations for low-dimensional Lie algebras, and introduce the concept of fixed basis vectors for proving that all almost inner derivations are inner for 2-step nilpotent Lie algebras determined by graphs, free 2 and 3-step nilpotent Lie algebras, free metabelian nilpotent Lie algebras on two generators, almost abelian Lie algebras and triangular Lie algebras. On the other hand we also exhibit families of nilpotent Lie algebras having an arbitrary large space of non-inner almost inner derivations.
\end{abstract}

\section{INTRODUCTION}

Almost inner automorphisms of Lie groups and almost inner derivations of Lie algebras have been introduced by Gordon and Wilson [7] in the study of isospectral deformations of compact solvmanifolds. A classical question going back to Hermann Weyl was whether or not isospectral manifolds are necessarily isometric. Milnor [10] in 1964 gave a negative answer by constructing two isospectral nonisometric flat tori in dimension 16. Mark Kac in 1966 gave the question the popular title "Can One Hear the Shape of a Drum?" The problem in two dimensions remained open until 1992, when Gordon, Webb, and Wolpert constructed, based on the Sunada method, a pair of regions in the plane that have different shapes but identical eigenspectra. In 1984 however, Gordon and Wilson wanted to construct not only finite families of isospectral nonisometric manifolds, but rather continuous families. They constructed isospectral but nonisometric compact Riemannian manifolds of the form $G / \Gamma$, with a simply connected exponential solvable Lie group $G$, and a discrete cocompact subgroup $\Gamma$ of $G$. For this construction, almost inner automorphisms and almost inner derivations were crucial.

The concept of "almost inner" automorphisms and derivations, almost homomorphisms, or almost conjugate subgroups arises in many contexts in algebra, number theory and geometry. Another example here is the study on the relation between element-conjugacy and global conjugacy for algebraic groups by Larsen [8]. This is, by a theorem of Sunada, closely related to the question of when a compact group can be the common covering space of a pair of non-isometric isospectral manifolds.

There are several other studies on related concepts, for example on local derivations [2], which are a generalization of almost inner derivations.

The goal of our paper is to begin a systematic study of almost inner derivations of Lie algebras. Gordon and Wilson, and later others have given several examples of solvable and nilpotent Lie algebras and their almost inner derivations. However, the methods were ad hoc, and many examples were restricted to 2-step nilpotent Lie algebras.

Date: September 17, 2018.

2010 Mathematics Subject Classification. 17B40.

Key words and phrases. Almost inner derivations, central almost inner derivations. 
The paper is structured as follows. We first introduce almost inner and central almost inner derivations, and prove basic properties. Then we give examples of Lie algebras in dimension 5 having non-inner almost inner derivations. In section 3 we explain the concept of fixed basis vectors. This enables us to show, without computing all derivations, that for several kinds of Lie algebras all almost inner derivations are already inner. This includes 2-step nilpotent Lie algebras determined by graphs, free 2-and 3-step nilpotent Lie algebras, free metabelian nilpotent Lie algebras on two generators, almost abelian Lie algebras and triangular Lie algebras. In section 7 we prove that all metabelian filiform Lie algebras of dimension $n \geq 5$, except for the standard graded one, admit a non-inner almost inner derivation. In section 8 we classify all complex Lie algebras of dimension 5 admitting a non-inner almost inner derivation, and compute the space of almost inner derivations for all complex nilpotent Lie algebras of dimension 6. Finally, we construct infinite families of Lie algebras $\mathfrak{g}$ having a space $\operatorname{AID}(\mathfrak{g}) / \operatorname{Inn}(\mathfrak{g})$ of arbitrarily large dimension $n$, for any given $n \in \mathbb{N}$.

\section{Preliminaries}

Unless otherwise specified all Lie algebras we consider are over a general field $K$. The definition of almost inner derivations of Lie algebras in [7] is as follows.

Definition 2.1. A derivation $D \in \operatorname{Der}(\mathfrak{g})$ of a Lie algebra $\mathfrak{g}$ is said to be almost inner, if $D(x) \in[\mathfrak{g}, x]$ for all $x \in \mathfrak{g}$. The space of all almost inner derivations of $\mathfrak{g}$ is denoted by $\operatorname{AID}(\mathfrak{g})$.

A derivation is almost inner if and only if it coincides on each one-dimensional subspace with an inner derivation. In particular, the set of all inner derivations $\operatorname{Inn}(\mathfrak{g})$ is a subset of $\operatorname{AID}(\mathfrak{g})$. Note that it is not enough in general to check the condition $D(x) \in[\mathfrak{g}, x]$ only for basis vectors of $\mathfrak{g}$. We introduce a new subspace of $\operatorname{AID}(\mathfrak{g})$ as follows.

Definition 2.2. An almost inner derivation $D \in \operatorname{AID}(\mathfrak{g})$ is called central almost inner if there exists an $x \in \mathfrak{g}$ such that $D-\operatorname{ad}(x)$ maps $\mathfrak{g}$ to the center $Z(\mathfrak{g})$. We denote the space of central almost inner derivations of $\mathfrak{g}$ by $\operatorname{CAID}(\mathfrak{g})$.

The subspaces $\operatorname{Inn}(\mathfrak{g}), \operatorname{CAID}(\mathfrak{g})$ and $\operatorname{AID}(\mathfrak{g})$ of $\operatorname{Der}(\mathfrak{g})$ become Lie subalgebras via the Lie bracket $\left[D, D^{\prime}\right]=D D^{\prime}-D^{\prime} D$.

Proposition 2.3. We have the following inclusions of Lie subalgebras

$$
\operatorname{Inn}(\mathfrak{g}) \subseteq \operatorname{CAID}(\mathfrak{g}) \subseteq \operatorname{AID}(\mathfrak{g}) \subseteq \operatorname{Der}(\mathfrak{g})
$$

Proof. Let $D, D^{\prime} \in \operatorname{AID}(\mathfrak{g})$ and $x \in \mathfrak{g}$. Then there exist $y, y^{\prime} \in \mathfrak{g}$ such that $D(x)=[y, x]$ and $D^{\prime}(x)=\left[y^{\prime}, x\right]$. Using the derivation rule and the Jacobi identity we obtain

$$
\begin{aligned}
{\left[D, D^{\prime}\right](x) } & =\left(D D^{\prime}\right)(x)-\left(D^{\prime} D\right)(x) \\
& =D\left(\left[y^{\prime}, x\right]\right)-D^{\prime}([y, x]) \\
& =\left[D\left(y^{\prime}\right), x\right]+\left[y^{\prime}, D(x)\right]-\left[D^{\prime}(y), x\right]-\left[y, D^{\prime}(x)\right] \\
& =\left[D\left(y^{\prime}\right), x\right]+\left[y^{\prime},[y, x]\right]-\left[D^{\prime}(y), x\right]-\left[y,\left[y^{\prime}, x\right]\right] \\
& =\left[D\left(y^{\prime}\right), x\right]-\left[\left[y, y^{\prime}\right], x\right]-\left[D^{\prime}(y), x\right] .
\end{aligned}
$$

Hence $\left[D, D^{\prime}\right](x)=\left[D\left(y^{\prime}\right)-\left[y, y^{\prime}\right]-D^{\prime}(y), x\right] \in[\mathfrak{g}, x]$ for all $x \in \mathfrak{g}$, so that $\left[D, D^{\prime}\right] \in \operatorname{AID}(\mathfrak{g})$. Let $C, C^{\prime} \in \operatorname{CAID}(\mathfrak{g})$. Then there exist $y, y^{\prime} \in \mathfrak{g} \operatorname{such}$ that $C-\operatorname{ad}(y)$ and $C^{\prime}-\operatorname{ad}\left(y^{\prime}\right) \operatorname{map} \mathfrak{g}$ to 
$Z(\mathfrak{g})$. Using $[D, \operatorname{ad}(x)]=\operatorname{ad}(D(x))$ for $D \in \operatorname{Der}(\mathfrak{g})$ we obtain

$$
\begin{aligned}
{\left[C-\operatorname{ad}(y), C^{\prime}-\operatorname{ad}\left(y^{\prime}\right)\right] } & =\left[C, C^{\prime}\right]-\left[C, \operatorname{ad}\left(y^{\prime}\right)\right]-\left[\operatorname{ad}(y), C^{\prime}\right]+\left[\operatorname{ad}(y), \operatorname{ad}\left(y^{\prime}\right)\right] \\
& =\left[C, C^{\prime}\right]-\operatorname{ad}\left(C\left(y^{\prime}\right)\right)+\operatorname{ad}\left(C^{\prime}(y)\right)+\operatorname{ad}\left(\left[y, y^{\prime}\right]\right),
\end{aligned}
$$

so that $\left[C, C^{\prime}\right]-\operatorname{ad}\left(C\left(y^{\prime}\right)-C^{\prime}(y)-\left[y, y^{\prime}\right]\right)=\left[C-\operatorname{ad}(y), C^{\prime}-\operatorname{ad}\left(y^{\prime}\right)\right] \operatorname{maps} \mathfrak{g}$ to $Z(\mathfrak{g})$, and hence $\left[C, C^{\prime}\right] \in \operatorname{CAID}(\mathfrak{g})$.

Proposition 2.4. The subalgebra $\operatorname{CAID}(\mathfrak{g})$ is a Lie ideal in $\operatorname{AID}(\mathfrak{g})$, and $\operatorname{Inn}(\mathfrak{g})$ is a Lie ideal in all subalgebras of $\operatorname{Der}(\mathfrak{g})$ containing it.

Proof. Let $C \in \mathrm{CAID}(\mathfrak{g})$ and $D \in \operatorname{AID}(\mathfrak{g})$. We need to show that $[D, C] \in \operatorname{CAID}(\mathfrak{g})$. We already know that $[D, C] \in \operatorname{AID}(\mathfrak{g})$. Fix an element $x \in \mathfrak{g}$ such that $C^{\prime}:=C-\operatorname{ad}(x)$ maps $\mathfrak{g}$ to $Z(\mathfrak{g})$. Define $D^{\prime}:=[D, C]-\operatorname{ad}(D(x))$. Then $D^{\prime}=\left[D, C^{\prime}\right]$ because of $\operatorname{ad}(D(x))=[D, \operatorname{ad}(x)]$, and $D^{\prime}$ maps $\mathfrak{g}$ to $Z(\mathfrak{g})$, since

$$
\begin{aligned}
D^{\prime}(y) & =\left[D, C^{\prime}\right](y) \\
& =D\left(C^{\prime}(y)\right)-C^{\prime}(D(y))
\end{aligned}
$$

for all $y \in \mathfrak{g}$, because $C^{\prime}$ maps $\mathfrak{g}$ to $Z(\mathfrak{g})$ and $D$ maps $Z(\mathfrak{g})$ to $Z(\mathfrak{g})$.

Finally, $[D, \operatorname{ad}(x)]=\operatorname{ad}(D(x))$ is inner for all $D \in \operatorname{Der}(\mathfrak{g})$, so that $\operatorname{Inn}(\mathfrak{g})$ is an ideal in all subalgebras of $\operatorname{Der}(\mathfrak{g})$ containing $\operatorname{Inn}(\mathfrak{g})$.

Remark 2.5. We conjecture that $\operatorname{AID}(\mathfrak{g})$ is always a Lie ideal in $\operatorname{Der}(\mathfrak{g})$. However, this seems not to be known, and there is no obvious algebraic argument for it.

As a first example we will compute the almost inner derivations of the Heisenberg Lie algebra $\mathfrak{n}_{3}(K)$, given by the Lie brackets $\left[e_{1}, e_{2}\right]=e_{3}$. By this notation we will mean that $\mathfrak{n}_{3}(K)$ is a 3 -dimensional vector space over $K$ with basis vectors $e_{1}, e_{2}$ and $e_{3}$. The Lie brackets between basis vectors which are not specified are assumed to be zero, so $\left[e_{1}, e_{3}\right]=\left[e_{2}, e_{3}\right]=0$.

Example 2.6. For $\mathfrak{g}=\mathfrak{n}_{3}(K)$ we have $\operatorname{AID}(\mathfrak{g})=\operatorname{Inn}(\mathfrak{g})$.

Indeed, every derivation $D$ of $\mathfrak{n}_{3}(K)$ is of the form $D\left(e_{1}\right)=\alpha_{1} e_{1}+\alpha_{2} e_{2}+\alpha_{3} e_{3}, D\left(e_{2}\right)=$ $\alpha_{4} e_{1}+\alpha_{5} e_{2}+\alpha_{6} e_{3}$, and $D\left(e_{3}\right)=\left(\alpha_{1}+\alpha_{5}\right) e_{3}$. Assume that $D$ is almost inner. Then $D\left(e_{1}\right) \in$ $\left[\mathfrak{g}, e_{1}\right]=\left\langle e_{3}\right\rangle$, so that $\alpha_{1}=\alpha_{2}=0$. In the same way $D\left(e_{2}\right) \in\left[\mathfrak{g}, e_{2}\right]=\left\langle e_{3}\right\rangle$ implies that $\alpha_{4}=\alpha_{5}=0$, and $D\left(e_{3}\right) \in\left[\mathfrak{g}, e_{3}\right]=0$ gives $\alpha_{1}+\alpha_{5}=0$. It follows that $D \in \operatorname{Inn}(\mathfrak{g})$.

The next examples show that there exist Lie algebras having more interesting almost inner derivations, i.e., having non-inner almost inner derivations. Let $\mathfrak{g}_{5,6}$ be the filiform nilpotent Lie algebra with basis $\left\{e_{1}, \ldots, e_{5}\right\}$ and Lie brackets

$$
\begin{aligned}
& {\left[e_{1}, e_{i}\right]=e_{i+1}, 2 \leq i \leq 4,} \\
& {\left[e_{2}, e_{3}\right]=e_{5} .}
\end{aligned}
$$

Let $\mathfrak{g}_{5,3}$ be the 3 -step nilpotent Lie algebra with basis $\left\{e_{1}, \ldots, e_{5}\right\}$ and Lie brackets

$$
\left[e_{1}, e_{2}\right]=e_{4},\left[e_{1}, e_{4}\right]=e_{5},\left[e_{2}, e_{3}\right]=e_{5}
$$

Denote by $E_{i j}$ the matrix with entry 1 at position $(i, j)$ and 0 otherwise. As a linear map, it maps $e_{k}$ to 0 for $k \neq j$, and $e_{j}$ to $e_{i}$.

Example 2.7. For $\mathfrak{g}=\mathfrak{g}_{5,6}$ we have $\operatorname{AID}(\mathfrak{g})=\operatorname{Inn}(\mathfrak{g}) \oplus\left\langle E_{5,2}\right\rangle$, and for $\mathfrak{g}=\mathfrak{g}_{5,3}$ we have $\operatorname{AID}(\mathfrak{g})=\operatorname{Inn}(\mathfrak{g}) \oplus\left\langle E_{5,3}\right\rangle$. 
The proof follows again by a direct computation. The derivation $D=E_{5,2}$ for $\mathfrak{g}_{5,6}$ is not inner, but almost inner (in fact, central almost inner). The same holds for $D=E_{5,3}$ for $\mathfrak{g}_{5,3}$. We will see later also examples of Lie algebras $\mathfrak{g}$ where the inclusion $\operatorname{CAID}(\mathfrak{g}) \subseteq \operatorname{AID}(\mathfrak{g})$ is strict.

The following table shows the result of a computation of almost inner derivations for all complex nilpotent Lie algebras of dimension 5. The classification of such Lie algebras is taken from [9]; $c(\mathfrak{g})$ denotes the nilpotency class of $\mathfrak{g}$, and $d(\mathfrak{g})$ the derived length. If the entry in the last column is non zero, it gives an example of an almost inner derivation which is not inner.

\begin{tabular}{c|c|c|c|c|c|c|c} 
Magnin & $c(\mathfrak{g})$ & $d(\mathfrak{g})$ & $\operatorname{dim} \operatorname{Inn}(\mathfrak{g})$ & $\operatorname{dim} \operatorname{CAID}(\mathfrak{g})$ & $\operatorname{dim} \operatorname{AID}(\mathfrak{g})$ & $\operatorname{dim} \operatorname{Der}(\mathfrak{g})$ & $D$ \\
\hline $\mathfrak{g}_{5,6}$ & 4 & 2 & 4 & 5 & 5 & 8 & $E_{5,2}$ \\
\hline $\mathfrak{g}_{5,5}$ & 4 & 2 & 4 & 4 & 4 & 9 & 0 \\
\hline $\mathfrak{g}_{5,3}$ & 3 & 2 & 4 & 5 & 5 & 10 & $E_{5,3}$ \\
\hline $\mathfrak{g}_{5,4}$ & 3 & 2 & 3 & 3 & 3 & 10 & 0 \\
\hline $\mathfrak{n}_{4} \oplus \mathbb{C}$ & 3 & 2 & 3 & 3 & 3 & 11 & 0 \\
\hline $\mathfrak{g}_{5,2}$ & 2 & 2 & 3 & 3 & 3 & 13 & 0 \\
\hline $\mathfrak{g}_{5,1}$ & 2 & 2 & 4 & 4 & 4 & 15 & 0 \\
\hline $\mathfrak{n}_{3} \oplus \mathbb{C}^{2}$ & 2 & 2 & 2 & 2 & 2 & 16 & 0 \\
\hline $\mathbb{C}^{5}$ & 1 & 1 & 0 & 0 & 0 & 25 & 0
\end{tabular}

A further computation shows that 5 is the minimal dimension where we have a complex Lie algebra admitting a non-inner almost inner derivation:

Proposition 2.8. Let $\mathfrak{g}$ be a complex Lie algebra of dimension $n \leq 4$. Then we have $\operatorname{AID}(\mathfrak{g})=$ $\operatorname{CAID}(\mathfrak{g})=\operatorname{Inn}(\mathfrak{g})$.

We will conclude this section with a few more easy facts on almost inner derivations. Clearly $\operatorname{Inn}(\mathfrak{g})=\operatorname{CAID}(\mathfrak{g})=\operatorname{AID}(\mathfrak{g})=0$ for abelian Lie algebras. Recall that a Lie algebra $\mathfrak{g}$ is called complete, if $Z(\mathfrak{g})=0$ and $\operatorname{Der}(\mathfrak{g})=\operatorname{Inn}(\mathfrak{g})$. Of course we have $\operatorname{AID}(\mathfrak{g})=\operatorname{CAID}(\mathfrak{g})=\operatorname{Inn}(\mathfrak{g})$ in this case. In particular semisimple Lie algebras, and parabolic subalgebras of semisimple Lie algebras are complete.

Proposition 2.9. Let $\mathfrak{g}$ be a Lie algebra. Then the following statements hold.

(1) Let $D \in \operatorname{AID}(\mathfrak{g})$. Then $D(\mathfrak{g}) \subseteq[\mathfrak{g}, \mathfrak{g}], D(Z(\mathfrak{g}))=0$ and $D(I) \subseteq$ I for every ideal I of $\mathfrak{g}$.

(2) For $D \in \mathrm{CAID}(\mathfrak{g})$ there exists an $x \in \mathfrak{g}$ such that $D_{\mid[\mathfrak{g}, \mathfrak{g}]}=\operatorname{ad}(x)_{\mid[\mathfrak{g}, \mathfrak{g}]}$.

(3) If $\mathfrak{g}$ is 2-step nilpotent, then $\operatorname{CAID}(\mathfrak{g})=\operatorname{AID}(\mathfrak{g})$.

(4) If $Z(\mathfrak{g})=0$, then $\operatorname{CAID}(\mathfrak{g})=\operatorname{Inn}(\mathfrak{g})$.

(5) If $\mathfrak{g}$ is nilpotent, then $\operatorname{AID}(\mathfrak{g})$ is nilpotent and all $D \in \operatorname{AID}(\mathfrak{g})$ are nilpotent.

(6) We have $\operatorname{AID}\left(\mathfrak{g} \oplus \mathfrak{g}^{\prime}\right)=\operatorname{AID}(\mathfrak{g}) \oplus \operatorname{AID}\left(\mathfrak{g}^{\prime}\right)$ for the direct sum of two Lie algebras.

Proof. By definition, an almost inner derivation maps $\mathfrak{g}$ into $[\mathfrak{g}, \mathfrak{g}]$ and the center $Z(\mathfrak{g})$ to 0 . Let $x \in I$. Then we have $D(x) \in[\mathfrak{g}, x] \subseteq[\mathfrak{g}, I] \subseteq I$, and (1) follows.

Given $D \in \operatorname{CAID}(\mathfrak{g})$, there exists an $x \in \mathfrak{g}$ such that $D^{\prime}=D-\operatorname{ad}(x)$ satisfies $D^{\prime}(\mathfrak{g}) \subseteq Z(\mathfrak{g})$. Since $D^{\prime}$ is also a derivation we have

$$
D^{\prime}([u, v])=\left[D^{\prime}(u), v\right]+\left[u, D^{\prime}(v)\right]=0
$$


for all $u, v \in \mathfrak{g}$. This shows (2). If $\mathfrak{g}$ is 2-step nilpotent then $D(\mathfrak{g}) \subseteq[\mathfrak{g}, \mathfrak{g}] \subseteq Z(\mathfrak{g})$ for any $D \in \operatorname{AID}(\mathfrak{g})$. Hence $\operatorname{AID}(\mathfrak{g}) \subseteq \operatorname{CAID}(\mathfrak{g})$, and we have equality. This shows (3). Suppose that $Z(\mathfrak{g})=0$ and $D \in \operatorname{CAID}(\mathfrak{g})$. Then there exists an $x \in \mathfrak{g}$ such that $D(x)-\operatorname{ad}(x)=0$. Hence $D$ is inner. This shows (4). Let $D \in \operatorname{AID}(\mathfrak{g})$ and $x \in \mathfrak{g}$, then $D^{k}(x) \in[\mathfrak{g},[\mathfrak{g},[\ldots,[\mathfrak{g}, x] \ldots]]]$ $\left(k\right.$ times $\mathfrak{g}$ ). If $k$ is higher than the nilpotence class of $\mathfrak{g}$, we have that $D^{k}(x)=0$, hence $D$ is nilpotent. By Engel's theorem $\operatorname{AID}(\mathfrak{g})$ is nilpotent, and (5) follows. For the last statement, let $D \in \operatorname{AID}\left(\mathfrak{g} \oplus \mathfrak{g}^{\prime}\right)$. Then the restrictions are again almost inner derivations, i.e., $D_{\mid \mathfrak{g}} \in \operatorname{AID}(\mathfrak{g})$ and $D_{\mid \mathfrak{g}^{\prime}} \in \operatorname{AID}\left(\mathfrak{g}^{\prime}\right)$. It is easy to see that the map $D \mapsto D_{\mid \mathfrak{g}} \oplus D_{\mid \mathfrak{g}^{\prime}}$ gives a one-to-one correspondence between $\operatorname{AID}\left(\mathfrak{g} \oplus \mathfrak{g}^{\prime}\right)$ and $\operatorname{AID}(\mathfrak{g}) \oplus \operatorname{AID}\left(\mathfrak{g}^{\prime}\right)$.

\section{FIXED BASIS VECTORS}

For the computation of almost inner derivations of a given Lie algebra one does not always need to know its derivation algebra explicitly. Instead one can use a concept, which we will call fixed basis vectors. This is very useful, also for proving several results on almost inner derivations. Unfortunately the definition is not particularly clear, although it is elementary. We will need to explain it with some examples.

For the rest of this section, $\mathfrak{g}$ is an $n$-dimensional Lie algebra over a field $K$ and with chosen basis $\left\{e_{1}, \ldots, e_{n}\right\}$. For $x \in \mathfrak{g}$ we denote the centralizer of $x$ by $C_{\mathfrak{g}}(x)=\{y \in \mathfrak{g} \mid[x, y]=0\}$. Let $D$ be an almost inner derivation $D$ of $\mathfrak{g}$. Then there exists a map $\varphi_{D}: \mathfrak{g} \rightarrow \mathfrak{g}$ such that

$$
D(x)=\left[x, \varphi_{D}(x)\right]
$$

for all $x \in \mathfrak{g}$. This map is not unique as we may change $\varphi_{D}(x)$ to $\varphi_{D}(x)+y$ for any $y \in C_{\mathfrak{g}}(x)$. It need also not be linear in general. If $x=\sum_{j=1}^{n} \alpha_{j} e_{j}$, then we denote by $t_{i}(x)=\alpha_{i}$ the $i$-th coordinate of $x$ with respect to the given basis.

Definition 3.1. Let $D$ be an almost inner derivation of $\mathfrak{g}$ determined by a map $\varphi_{D}: \mathfrak{g} \rightarrow \mathfrak{g}$. We will say that a basis vector $e_{i}$ is a fixed vector for $D$ with fixed value $\alpha \in K$ if and only if for all $j \in\{1,2, \ldots n\}$ :

$$
\text { if } e_{j} \notin C_{\mathfrak{g}}\left(e_{i}\right) \text { then } t_{i}\left(\varphi_{D}\left(e_{j}\right)\right)=\alpha \text {. }
$$

Note that the $\alpha$ must be the same for all $j$ where this condition applies. As an example, consider the Heisenberg Lie algebra $\mathfrak{n}_{3}(K)$ with basis $\left\{e_{1}, e_{2}, e_{3}\right\}$ and Lie bracket $\left[e_{1}, e_{2}\right]=e_{3}$.

Example 3.2. Let $\mathfrak{g}=\mathfrak{n}_{3}(K)$, and $D$ an almost inner derivation of $\mathfrak{g}$ given by a map $\varphi_{D}: \mathfrak{g} \rightarrow$ $\mathfrak{g}$. Then every basis vector $e_{i}$ is fixed.

For $i=1$ we have $C_{\mathfrak{g}}\left(e_{1}\right)=\left\langle e_{1}, e_{3}\right\rangle$, and the condition just applies for $j=2$ : since $e_{2} \notin C_{\mathfrak{g}}\left(e_{1}\right)$, we must have $t_{1}\left(\varphi_{D}\left(e_{2}\right)\right)=\alpha$. Certainly this is true, with the $\alpha$ given by the map $\varphi_{D}$. The same holds for $i=2$, where we have $C_{\mathfrak{g}}\left(e_{2}\right)=\left\langle e_{2}, e_{3}\right\rangle$. For $i=3$ we have $C_{\mathfrak{g}}\left(e_{3}\right)=\mathfrak{g}$, so that the condition is vacuously true.

The importance of finding fixed vectors comes from the following fact. If each basis vector for every almost inner derivations is fixed, then we have $\operatorname{AID}(\mathfrak{g})=\operatorname{Inn}(\mathfrak{g})$. We will prove this result in Corollary 3.6. Often we can show that every basis vector is fixed without knowing the structure of $\operatorname{Der}(\mathfrak{g})$. A trivial example is the following lemma.

Lemma 3.3. Let $\mathfrak{g}$ be a Lie algebra with given basis $\left\{e_{1}, \ldots, e_{n}\right\}$, such that for given $i$, the number of basis vectors in $C_{\mathfrak{g}}\left(x_{i}\right)$ is equal to $\operatorname{dim}(\mathfrak{g})$ or $\operatorname{dim}(\mathfrak{g})-1$. Then the basis vector $e_{i}$ is fixed. 
Proof. In this case the condition for a fixed basis vector is vacuously true, or can be satisfied uniquely by the $\alpha$ given by the map $\varphi_{D}$.

We already saw this argument for $i=1,2,3$ in the example of $\mathfrak{g}=\mathfrak{n}_{3}(K)$ above.

We also want to present an example, where not every basis vector is fixed. For the Lie algebra $\mathfrak{g}_{5,6}$ of Example 2.7 we will show that there is an almost inner derivation $D$ determined by a map $\varphi_{D}$ such that not every basis vector is fixed.

Example 3.4. For $\mathfrak{g}=\mathfrak{g}_{5,6}$ and the almost inner derivation $D=E_{5,2}$ the basis vector $e_{3}$ is not fixed.

We need to find a map $\varphi_{D}$ representing $D$. Let $x=\alpha_{1} e_{1}+\ldots+\alpha_{5} e_{5} \in \mathfrak{g}$. Define $\varphi_{D}$ as follows:

(1) If $\alpha_{1} \neq 0$, then $\varphi_{D}(x)=\frac{\alpha_{2}}{\alpha_{1}} e_{4}$.

(2) If $\alpha_{1}=0$, then $\varphi_{D}(x)=e_{3}$.

It is easy to see that $D(x)=\left[x, \varphi_{D}(x)\right]$ for all $x \in \mathfrak{g}$. Definition 3.1 for this $\varphi_{D}$ and $i=3$ says: for all $j \in\{1, \ldots, 5\}$, if $e_{j} \notin C_{\mathfrak{g}}\left(e_{3}\right)=\left\langle e_{3}, e_{4}, e_{5}\right\rangle$, then $t_{3}\left(\varphi_{D}\left(e_{j}\right)\right)=\alpha$, each time for the same fixed $\alpha$. This applies for $j=1,2$, and we have $\varphi_{D}\left(e_{1}\right)=0, \varphi_{D}\left(e_{2}\right)=e_{3}$, so that

$$
\begin{aligned}
& t_{3}\left(\varphi_{D}\left(e_{2}\right)\right)=t_{3}\left(e_{3}\right)=1, \\
& t_{3}\left(\varphi_{D}\left(e_{1}\right)\right)=t_{3}(0)=0 .
\end{aligned}
$$

So there is no fixed $\alpha$, and $e_{3}$ is not fixed.

Lemma 3.5. Let $D: \mathfrak{g} \rightarrow \mathfrak{g}$ be an almost inner derivation determined by a map $\varphi_{D}: \mathfrak{g} \rightarrow \mathfrak{g}$. If $e_{i}$ is a fixed basis vector with fixed value $\alpha$, then $D^{\prime}=D+\operatorname{ad}\left(\alpha e_{i}\right)$ is an almost inner derivation which is determined by a map $\varphi_{D^{\prime}}: \mathfrak{g} \rightarrow \mathfrak{g}$ such that for all $j, k \in\{1,2, \ldots, n\}$ :

$$
\begin{aligned}
& t_{j}\left(\varphi_{D^{\prime}}\left(e_{k}\right)\right)=t_{j}\left(\varphi_{D}\left(e_{k}\right)\right) \text { for } i \neq j \\
& t_{i}\left(\varphi_{D^{\prime}}\left(e_{k}\right)\right)=0 .
\end{aligned}
$$

Proof. Clearly $D^{\prime}$ is an almost inner derivation, and we have that

$$
\left(D+\operatorname{ad}\left(\alpha e_{i}\right)\right)(x)=\left[x, \varphi_{D}(x)\right]+\left[\alpha e_{i}, x\right]=\left[x, \varphi_{D}(x)-\alpha e_{i}\right] .
$$

So $D^{\prime}$ is determined by the map $\tilde{\varphi}_{D^{\prime}}: \mathfrak{g} \rightarrow \mathfrak{g}: x \mapsto \varphi_{D}(x)-\alpha e_{i}$.

Now define the map

$$
\varphi_{D^{\prime}}: \mathfrak{g} \rightarrow \mathfrak{g}: x \mapsto \begin{cases}\varphi_{D}(x)-\alpha e_{i} & \text { if } x \notin\left\{e_{1}, e_{2}, \ldots, e_{n}\right\} \\ \varphi_{D}(x)-t_{i}\left(\varphi_{D}(x)\right) e_{i} & \text { if } x \in\left\{e_{1}, e_{2}, \ldots, e_{n}\right\}\end{cases}
$$

We claim that $D^{\prime}$ is also determined by this new map $\varphi_{D^{\prime}}$. Indeed, for all non basis vectors we have $\varphi_{D^{\prime}}(x)=\tilde{\varphi}_{D^{\prime}}(x)$, so we only have to consider basis vectors. Let $e_{j}$ be a basis vector. Then there are two possibilities:

Case 1: $e_{j} \in C_{\mathfrak{g}}\left(e_{i}\right)$. Then we have

$$
D^{\prime}\left(e_{j}\right)=D\left(e_{j}\right)=\left[e_{j}, \varphi_{D}\left(e_{j}\right)\right]=\left[e_{j}, \varphi_{D}\left(e_{j}\right)-t_{i}\left(\varphi_{D}(x)\right) e_{i}\right]=\left[e_{j}, \varphi_{D^{\prime}}\left(e_{j}\right)\right] .
$$

Case 2: $e_{j} \notin C_{\mathfrak{g}}\left(e_{i}\right)$. Then $t_{i}\left(\varphi_{D}\left(e_{j}\right)\right)=\alpha$, from which it follows that $\tilde{\varphi}_{D^{\prime}}\left(e_{j}\right)=\varphi_{D^{\prime}}\left(e_{j}\right)$.

Hence $D^{\prime}$ is determined by $\varphi_{D^{\prime}}$. By definition of $\varphi_{D^{\prime}}$ it is also easy to see that the requirements $t_{j}\left(\varphi_{D^{\prime}}\left(e_{k}\right)\right)=t_{j}\left(\varphi_{D}\left(e_{k}\right)\right)$, for $j \neq i$, and $t_{i}\left(\varphi_{D^{\prime}}\left(e_{k}\right)\right)=0$ hold. 
As an immediate consequence we obtain the following result.

Corollary 3.6. Let $D \in \mathrm{AID}(\mathfrak{g})$ be determined by a map $\varphi_{D}$. If each basis vector is fixed, then $D \in \operatorname{Inn}(\mathfrak{g})$.

Proof. Let $\alpha_{i}$ denote the fixed value of $e_{i}$. Then by iteratively applying Lemma 3.5, we find that $D+\operatorname{ad}\left(\alpha_{1} e_{1}\right)+\operatorname{ad}\left(\alpha_{2} e_{2}\right)+\cdots+\operatorname{ad}\left(\alpha_{n} e_{n}\right)=D+\operatorname{ad}(v)$, with $v=\sum_{i=1}^{n} \alpha_{i} e_{i}$ is an almost inner derivation $D^{\prime}$, determined by a map $\varphi_{D^{\prime}}$ with $\varphi_{D^{\prime}}\left(e_{i}\right)=0$ for all $i \in\{1,2, \ldots, n\}$. This implies that $D^{\prime}\left(e_{i}\right)=0$ for all basis vectors $e_{i}$ and hence $D^{\prime}=0$ or $D=-\operatorname{ad}(v) \in \operatorname{Inn}(\mathfrak{g})$.

The next results are two technical lemmas, providing a way to find fixed basis vectors. We will use the following notation: Let $i_{1}, i_{2}, \ldots, i_{r} \in\{1,2, \ldots, n\}$ then

$$
\mathfrak{g}_{i_{1}, i_{2}, \ldots, i_{r}}=\operatorname{span}\left\{e_{i} \mid i \notin\left\{i_{1}, i_{2}, \ldots, i_{r}\right\}\right\}
$$

denotes the vector space spanned by all basis vectors not in the set $\left\{e_{i_{1}}, e_{i_{2}}, \ldots, e_{i_{r}}\right\}$.

Lemma 3.7. Assume that $1 \leq i, j, k, l, m \leq n$ and $l \neq m$. Moreover assume that there exist nonzero scalars $\alpha, \beta \in K$ such that

$$
\begin{aligned}
& {\left[e_{j}, e_{i}\right]-\alpha e_{l} \in \mathfrak{g}_{l, m}} \\
& {\left[e_{k}, e_{i}\right]-\beta e_{m} \in \mathfrak{g}_{l, m}} \\
& {\left[e_{j}, \mathfrak{g}_{i}\right] \subseteq \mathfrak{g}_{l, m}} \\
& {\left[e_{k}, \mathfrak{g}_{i}\right] \subseteq \mathfrak{g}_{l, m} .}
\end{aligned}
$$

Then, for any $D \in \mathrm{AID}(\mathfrak{g})$ determined by a map $\varphi_{D}$, we have that $t_{i}\left(\varphi_{D}\left(e_{j}\right)\right)=t_{i}\left(\varphi_{D}\left(e_{k}\right)\right)$.

Proof. Let $a=t_{i}\left(\varphi_{D}\left(e_{j}\right)\right), b=t_{i}\left(\varphi_{D}\left(e_{k}\right)\right)$ and $c=t_{i}\left(\varphi_{D}\left(e_{j}+e_{k}\right)\right)$. Then there exist vectors $v, v^{\prime}, v^{\prime \prime} \in \mathfrak{g}_{i}$ such that

$$
\begin{aligned}
\varphi_{D}\left(e_{j}\right) & =a e_{i}+v, \\
\varphi_{D}\left(e_{k}\right) & =b e_{i}+v^{\prime}, \\
\varphi_{D}\left(e_{j}+e_{k}\right) & =c e_{i}+v^{\prime \prime} .
\end{aligned}
$$

Using these notations we find that

$$
D\left(e_{j}+e_{k}\right)=\left[e_{j}+e_{k}, c e_{i}+v^{\prime \prime}\right]=c \alpha e_{l}+c \beta e_{m}+w^{\prime \prime}
$$

for some $w^{\prime \prime} \in \mathfrak{g}_{l, m}$, and on the other hand we have that

$$
D\left(e_{j}\right)+D\left(e_{k}\right)=\left[e_{j}, a e_{i}+v\right]+\left[e_{k}, b e_{i}+v^{\prime}\right]=a \alpha e_{l}+w+b \beta e_{m}+w^{\prime}
$$

for some $w, w^{\prime} \in \mathfrak{g}_{l, m}$. Now, as $D$ is a linear map, the two expressions (11) and (2) must be equal, and so by comparing the $l$-th and $m$-th coordinate, we find that

$$
c \alpha=a \alpha, c \beta=b \beta .
$$

As both $\alpha$ and $\beta$ are nonzero this implies that $a=b$ and hence

$$
t_{i}\left(\varphi_{D}\left(e_{j}\right)\right)=t_{i}\left(\varphi_{D}\left(e_{k}\right)\right) .
$$


Lemma 3.8. Assume that $1 \leq i, j, k, l \leq n$. Moreover assume that there exist nonzero scalars $\alpha, \beta \in K$ such that

$$
\begin{aligned}
& {\left[e_{j}, e_{i}\right]-\alpha e_{l} \in \mathfrak{g}_{l}} \\
& {\left[e_{k}, e_{i}\right]-\beta e_{l} \in \mathfrak{g}_{l}} \\
& {\left[e_{j}, \mathfrak{g}_{i}\right] \subseteq \mathfrak{g}_{l}} \\
& {\left[e_{k}, \mathfrak{g}_{i}\right] \subseteq \mathfrak{g}_{l} .}
\end{aligned}
$$

Then, for any $D \in \operatorname{AID}(\mathfrak{g})$ determined by a map $\varphi_{D}$, we have that $t_{i}\left(\varphi_{D}\left(e_{j}\right)\right)=t_{i}\left(\varphi_{D}\left(e_{k}\right)\right)$.

Proof. Let $a=t_{i}\left(\varphi_{D}\left(e_{j}\right)\right), b=t_{i}\left(\varphi_{D}\left(e_{k}\right)\right)$ and $c=t_{i}\left(\varphi_{D}\left(\beta e_{j}-\alpha e_{k}\right)\right)$. Let $v, v^{\prime}, v^{\prime \prime} \in \mathfrak{g}_{i}$ be such that

$$
\begin{aligned}
\varphi_{D}\left(e_{j}\right) & =a e_{i}+v, \\
\varphi_{D}\left(e_{k}\right) & =b e_{i}+v^{\prime} \\
\varphi_{D}\left(\beta e_{j}-\alpha e_{k}\right) & =c e_{i}+v^{\prime \prime} .
\end{aligned}
$$

Then we have that

$$
D\left(\beta e_{j}-\alpha e_{k}\right)=\left[\beta e_{j}-\alpha e_{k}, c e_{i}+v^{\prime \prime}\right]=\beta c \alpha e_{l}-\alpha c \beta e_{l}+w^{\prime \prime}
$$

for some $w^{\prime \prime} \in \mathfrak{g}_{l}$. On the other hand we have that

$$
\beta D\left(e_{j}\right)-\alpha D\left(e_{k}\right)=\beta\left[e_{j}, a e_{i}+v\right]-\alpha\left[e_{k}, b e_{i}+v^{\prime}\right]=\beta a \alpha e_{l}+w-\alpha b \beta e_{l}+w^{\prime}
$$

for some $w, w^{\prime} \in \mathfrak{g}_{l}$. By comparing the $l$-th coordinate of (3) and (41) we find that

$$
\alpha \beta(a-b)=0 \Rightarrow a=b .
$$

\section{2-STEP NILPOTENT LIE ALGEBRAS DETERMINED BY GRAPHS}

Let $G(V, E)$ be a finite simple graph with $V=\left\{x_{1}, x_{2}, \ldots, x_{r}\right\}$ its set of vertices and $E$ its set of edges. If there is an edge between vertex $x_{i}$ and $x_{j}$ with $i<j$, we denote this edge by the symbol $y_{i, j}$. We let $X$ be the vector space over the field $K$ with basis the elements of $V$ and $Y$ be the vector space with basis the edges $y_{i, j}$. We define a two-step nilpotent Lie algebra $\mathfrak{g}$ over $K$, where as a vector space $\mathfrak{g}=X \oplus Y$ and where the brackets are given by

$$
\begin{aligned}
{\left[x_{i}, x_{j}\right] } & =\left\{\begin{array}{l}
y_{i, j}, \text { if } y_{i, j} \in E \\
0, \text { if there is no edge connecting } x_{i} \text { with } x_{j}
\end{array}\right. \\
{\left[x_{i}, y_{j, k}\right] } & =0 \quad \forall x_{i} \in V, \forall y_{j, k} \in E \\
{\left[y_{i, j}, y_{k, l}\right] } & =0 \quad \forall y_{i, j}, y_{k, l} \in E
\end{aligned}
$$

Theorem 4.1. Let $\mathfrak{g}$ be a 2-step nilpotent Lie algebra determined by a finite simple graph. Then $\operatorname{AID}(\mathfrak{g})=\operatorname{Inn}(\mathfrak{g})$.

Proof. Let $s=\# E$ and choose an order $p_{1}, p_{2}, \ldots p_{s}$ for the edges. So any $p_{t}$ corresponds to a unique edge $y_{i, j}$. Now, we fix the basis $\left\{e_{1}, e_{2}, \ldots, e_{r+s}\right\}$ of $\mathfrak{g}$ given by

$$
e_{1}=x_{1}, \ldots, e_{r}=x_{r}, e_{r+1}=p_{1}, e_{r+2}=p_{2}, \ldots, e_{r+s}=p_{s} .
$$

Let $D \in \operatorname{AID}(\mathfrak{g})$ be determined by the map $\varphi_{D}$. We want to apply Corollary 3.6, and hence we want to show that any basis vector is fixed for $D$. For $e_{r+1}, e_{r+2}, \ldots, e_{r+s}$ this is obvious, since these vectors belong to $Z(\mathfrak{g})$. 
Now, consider $e_{i}$ with $1 \leq i \leq r$. If $e_{i} \in Z(\mathfrak{g})$, i.e., when $x_{i}$ is an isolated vertex, there is again nothing to show. So assume that $e_{i} \notin Z(\mathfrak{g})$. Then there is at least one $e_{j} \notin C_{\mathfrak{g}}\left(e_{i}\right)$ (with $1 \leq j \leq n)$. Hence $\left[e_{j}, e_{i}\right]= \pm e_{l}$ for some $l$ between $r+1$ and $r+s$. Let $\alpha=t_{i}\left(\varphi_{D}\left(e_{j}\right)\right)$. Consider any other basis vector $e_{k} \notin C_{\mathfrak{g}}\left(e_{i}\right)$. In order to show that $e_{i}$ is fixed, we must show that also $t_{i}\left(\varphi_{D}\left(e_{k}\right)\right)=\alpha$. There exists an $m \in\{r+1, \ldots, r+s\}$ with $\left[e_{k}, e_{i}\right]= \pm e_{m}$. As $\mathfrak{g}$ is determined by a graph we have that $m \neq l$.

We are in the following situation

$$
\begin{aligned}
& {\left[e_{j}, e_{i}\right] \pm e_{l}=0} \\
& {\left[e_{k}, e_{i}\right] \pm e_{m}=0} \\
& {\left[e_{j}, \mathfrak{g}_{i}\right] \subseteq \mathfrak{g}_{l, m}} \\
& {\left[e_{k}, \mathfrak{g}_{i}\right] \subseteq \mathfrak{g}_{l, m} .}
\end{aligned}
$$

This means that we can apply Lemma 3.7 and we find that $t_{i}\left(\varphi_{D}\left(e_{k}\right)\right)=t_{i}\left(\varphi_{D}\left(e_{j}\right)\right)=\alpha$. Hence $e_{i}$ is indeed fixed for all $i$ and this finishes the proof.

Corollary 4.2. Let $\mathfrak{f}_{r, 2}$ be the free 2-step nilpotent Lie algebra on $r$ generators, then

$$
\operatorname{AID}\left(\mathfrak{f}_{r, 2}\right)=\operatorname{Inn}\left(\mathfrak{f}_{r, 2}\right) .
$$

Proof. This follows immediately from the fact that $\mathfrak{f}_{r, 2}$ is the 2-step nilpotent Lie algebra determined by the complete graph on $r$ vertices.

\section{Free 3-Step nilpotent Lie Algebras}

Let $\mathfrak{f}_{r, 3}$ be the free 3-step nilpotent Lie algebra on $r$ generators $e_{1}, e_{2}, \ldots, e_{r}$. Having fixed these generators, we can find a Hall basis of $\mathfrak{f}_{r, 3}$, which is a basis of $\mathfrak{f}_{r, 3}$ as a vector space and which is explicitly given by the following collection of vectors:

$$
\begin{aligned}
e_{i} & \text { for } 1 \leq i \leq r \\
y_{i, j} & =\left[e_{i}, e_{j}\right] \text { for } 1 \leq i<j \leq r \\
z_{i, j, k} & =\left[e_{i}, y_{j, k}\right] \text { for } 1 \leq j<k \leq r \text { and } 1 \leq i \leq k .
\end{aligned}
$$

Note that if $i>k$ then

$$
\begin{aligned}
{\left[e_{i}, y_{j, k}\right] } & =\left[e_{i},\left[e_{j}, e_{k}\right]\right] \\
& =-\left[e_{j},\left[e_{k}, e_{i}\right]\right]-\left[e_{k},\left[e_{i}, e_{j}\right]\right] \\
& =-z_{j, k, i}+z_{k, j, i} .
\end{aligned}
$$

Lemma 5.1. Let $x, y \in \mathfrak{f}_{r, 3}$. If $x-y \notin\left[\mathfrak{f}_{r, 3}, \mathfrak{f}_{r, 3}\right]$, then

$$
\left[x,\left[\mathfrak{f}_{r, 3}, \mathfrak{f}_{r, 3}\right]\right] \cap\left[y,\left[\mathfrak{f}_{r, 3}, \mathfrak{f}_{r, 3}\right]\right]=0 .
$$

Proof. If either $x$ or $y$ belongs to $\left[\mathfrak{f}_{r, 3}, \mathfrak{f}_{r, 3}\right]$ there is nothing to show. In case both do not belong to $\left[\mathfrak{f}_{r, 3}, \mathfrak{f}_{r, 3}\right]$, the condition that $x-y \notin\left[\mathfrak{f}_{r, 3}, \mathfrak{f}_{r, 3}\right]$, actually means that we can choose a generating set $e_{1}=x, e_{2}=y, e_{3}, \ldots, e_{r}$ such that $\mathfrak{f}_{r, 3}$ is the free 3-step nilpotent Lie algebra on that set of generators. Using the Hall basis introduced above, we see that

$$
\begin{aligned}
& {\left[x,\left[\mathfrak{f}_{r, 3}, \mathfrak{f}_{r, 3}\right]\right]=\left\langle z_{1, p, q} \mid 1 \leq p<q \leq r\right\rangle,} \\
& {\left[y,\left[\mathfrak{f}_{r, 3}, \mathfrak{f}_{r, 3}\right]\right]=\left\langle z_{2, p, q} \mid 1 \leq p<q \leq r\right\rangle .}
\end{aligned}
$$


Note that all of the vectors $z_{1, p, q}$ and $z_{2, p, q}$ belong to the Hall set mentioned above and that the set of basis vectors $z_{1, p, q}$ is disjoint of the set of basis vectors $z_{2, p, q}$. So we have that the subspaces spanned by those two sets have only the zero vector in common.

Theorem 5.2. Let $f_{r, 3}$ be the free 3-step nilpotent Lie algebra on $r$ generators. Then

$$
\operatorname{AID}\left(\mathfrak{f}_{r, 3}\right)=\operatorname{Inn}\left(\mathfrak{f}_{r, 3}\right) .
$$

Proof. Let $D \in \operatorname{AID}\left(\mathfrak{f}_{r, 3}\right)$. Note that $D$ induces an almost inner derivation $\bar{D}$ on $\mathfrak{f}_{r, 3} / Z\left(\mathfrak{f}_{r, 3}\right) \cong$ $\mathfrak{f}_{r, 2}$. By Corollary 4.2 we know that $\bar{D}$ is an inner derivation. Hence, by adjusting $D$ with an inner derivation, we may assume that $D\left(\mathfrak{f}_{r, 3}\right) \subseteq Z\left(\mathfrak{f}_{r, 3}\right)$.

Let $e_{1}, e_{2}, \ldots, e_{r}$ be the generators of $\mathfrak{f}_{r, 3}$. Since we must have that $D\left(e_{i}\right) \in Z\left(\mathfrak{f}_{r, 3}\right)$, there exist vectors $v_{i} \in\left[\mathfrak{f}_{r, 3}, \mathfrak{f}_{r, 3}\right]$ such that

$$
D\left(e_{i}\right)=\left[e_{i}, v_{i}\right]
$$

Analogously, there are also vectors $w_{i} \in\left[\mathfrak{f}_{r, 3}, \mathfrak{f}_{r, 3}\right]$, for $2 \leq i \leq r$, with

$$
D\left(e_{1}+e_{i}\right)=\left[e_{1}+e_{i}, w_{i}\right] .
$$

By using the equation $D\left(e_{1}+e_{i}\right)=D\left(e_{1}\right)+D\left(e_{i}\right)$ we find that

$$
\left[e_{1}, w_{i}\right]-\left[e_{1}, v_{1}\right]=\left[e_{i}, v_{i}\right]-\left[e_{i}, w_{i}\right] .
$$

Now, since the left hand side of the above expression belongs to $\left[e_{1},\left[\mathfrak{f}_{r, 3}, \mathfrak{f}_{r, 3}\right]\right]$ and the right hand side to $\left[e_{i},\left[\mathfrak{f}_{r, 3}, \mathfrak{f}_{r, 3}\right]\right]$, it follows from Lemma 5.1 that both expressions are zero. Hence we have

$$
\left[e_{1}, w_{i}-v_{1}\right]=\left[e_{i}, w_{i}-v_{i}\right]=0 .
$$

Since the only elements of $\left[\mathfrak{f}_{r, 3}, \mathfrak{f}_{r, 3}\right]$ that commute with $e_{1}$, respectively with $e_{i}$, are those belonging to the center $Z\left(\mathfrak{f}_{r, 3}\right)$, we find that

$$
w_{i}-v_{1} \in Z\left(\mathfrak{f}_{r, 3}\right), w_{i}-v_{i} \in Z\left(\mathfrak{f}_{r, 3}\right) .
$$

So $v_{i}-v_{1} \in Z\left(\mathfrak{f}_{r, 3}\right)$. Therefore we can without any problem replace $v_{i}$ with $v_{1}$, and we find that $D\left(e_{i}\right)=\left[e_{i}, v_{1}\right]$. If we now consider the derivation $D^{\prime}=D+\operatorname{ad}\left(v_{1}\right)$, we see that $D^{\prime}\left(e_{i}\right)=0$. But then $D^{\prime}$ is a derivation which is zero on the generators, and hence $D^{\prime}$ is zero everywhere. It follows that $D=-\operatorname{ad}\left(v_{1}\right)$, which was to be shown.

\section{Free metabelian nilpotent Lie algebras on tWo generators}

In this section we will show that all almost inner derivations are inner for free metabelian nilpotent Lie algebras of class $c$ on 2 generators.

Let $\mathfrak{f}_{2}$ be the free Lie algebra on two generators, say $a$ and $b$. Let $\mathfrak{f}_{2}^{(1)}=\left[\mathfrak{f}_{2}, \mathfrak{f}_{2}\right], \mathfrak{f}_{2}^{(i+1)}=\left[\mathfrak{f}_{2}^{(i)}, \mathfrak{f}_{2}^{(i)}\right]$ for $i \geq 1$, and $\gamma_{1}\left(\mathfrak{f}_{2}\right)=\mathfrak{f}_{2}, \gamma_{i+1}\left(\mathfrak{f}_{2}\right)=\left[\mathfrak{f}_{2}, \gamma_{i}\left(\mathfrak{f}_{2}\right)\right]$ for $i \geq 1$. Then, the free $c$-step nilpotent and metabelian Lie algebra $\mathfrak{m}_{2, c}$ is obtained as a quotient

$$
\mathfrak{m}_{2, c}=\frac{\mathfrak{f}_{2}}{\mathfrak{f}_{2}^{(2)}+\gamma_{c+1}\left(\mathfrak{f}_{2}\right)} .
$$

So, it is the largest quotient of $\mathfrak{f}_{2}$ which is both metabelian and $c$-step nilpotent. Let us use $x_{1}$ and $x_{2}$ to denote the projection of $a$ and $b$ resp. in $\mathfrak{m}_{2, c}$. We introduce the notation $y_{n}^{m}$ for all $m \geq 2, n \in\{1, \ldots, m-1\}$ by

$$
y_{n}^{m}=[x_{2}, \underbrace{x_{1}, x_{1}, x_{1}, \ldots, x_{1}}_{m-n \text { times }}, \underbrace{x_{2}, x_{2}, x_{2}, \ldots, x_{2}}_{n-1 \text { times }}] \text {, }
$$


where for all $z_{1}, z_{2}, \ldots, z_{n} \in \mathfrak{g}$, the iterated bracket $\left[\left[\ldots\left[\left[z_{1}, z_{2}\right], z_{3}\right], \ldots\right], z_{n}\right]$ is denoted with $\left[z_{1}, z_{2}, \ldots, z_{n}\right]$. So $y_{n}^{m}$ is an $m$-fold Lie bracket with $m-n$ appearances of $x_{1}$ and $n$ appearances of $x_{2}$. It is well known that $x_{1}, x_{2}$ together with the elements $y_{n}^{m}(1 \leq n<m \leq c)$ form a basis of $\mathfrak{m}_{2, c}$ (E.g. [3, Section 4.7]). In fact, for any $i>1$ the projections of the elements $y_{1}^{i}, y_{2}^{i}, \ldots, y_{i-1}^{i}$ form a basis of $\gamma_{i}\left(\mathfrak{m}_{2, c}\right) / \gamma_{i+1}\left(\mathfrak{m}_{2, c}\right)$. So $\gamma_{i}\left(\mathfrak{m}_{2, c}\right) / \gamma_{i+1}\left(\mathfrak{m}_{2, c}\right)$ is $(i-1)$-dimensional (for $i \leq c)$.

Lemma 6.1. Let $z_{1}, z_{2}, \ldots, z_{n-2} \in\left\{x_{1}, x_{2}\right\}$ and $k=\#\left\{i \in\{1, \ldots, n-2\} \mid z_{i}=x_{2}\right\}+1$. Then we have

$$
\left[x_{2}, x_{1}, z_{1}, z_{2}, \ldots, z_{n-2}\right]=y_{k}^{n} .
$$

Proof. In a metabelian Lie algebra $\mathfrak{g}$, it follows from the Jacobi identity that

$$
\forall x, y \in \mathfrak{g}, \forall c \in \gamma_{2}(\mathfrak{g}):[c, x, y]=[c, y, x] .
$$

From this it follows that

$$
\left[x_{2}, x_{1}, z_{1}, z_{2}, \ldots, z_{n-2}\right]=\left[x_{2}, x_{1}, z_{\sigma(1)}, z_{\sigma(2)}, \ldots, z_{\sigma(n-2)}\right]
$$

for any permutation $\sigma$ on $n-2$ letters $1,2, \ldots, n-2$. Now, the result follows easily.

The lemma easily implies the following identities.

Corollary 6.2. We have

$$
\left[y_{n}^{m}, x_{1}\right]=y_{n}^{m+1} \text { and }\left[y_{n}^{m}, x_{2}\right]=y_{n+1}^{m+1} .
$$

Now we can prove the main result of this section.

Proposition 6.3. Let $\mathfrak{m}_{2, c}$ be the free c-step nilpotent and metabelian Lie algebra on 2 generators over an infinite field $K$. Then $\operatorname{AID}\left(\mathfrak{m}_{2, c}\right)=\operatorname{Inn}\left(\mathfrak{m}_{2, c}\right)$.

Proof. For $c=1$ we have that $\mathfrak{m}_{2, c}$ is abelian and for $c=2$ we have that $\mathfrak{m}_{2, c}$ is the Heisenberg Lie algebra. As these Lie algebras have no non-trivial almost inner derivations the proposition is valid in this situation.

For general $c \geq 3$, we proceed by induction and so we assume the proposition holds up to $c-1$. Let $D$ be an almost inner derivation of $\mathfrak{m}_{2, c}$. The space $I=\left\langle y_{1}^{c}, y_{2}^{c}, \ldots, y_{c-1}^{c}\right\rangle=\gamma_{c}\left(\mathfrak{m}_{2, c}\right)=$ $Z\left(\mathfrak{m}_{2, c}\right)$ is an ideal of $\mathfrak{m}_{2, c}$ and hence $D$ induces an almost inner derivation $\bar{D}$ on

$$
\mathfrak{m}_{2, c} / I \cong \mathfrak{m}_{2, c-1} \text {. }
$$

By the induction hypothesis, $\bar{D}$ is an inner derivation of $\mathfrak{m}_{2, c-1}$. This means that we can alter $D$ by an inner derivation of $\mathfrak{m}_{2, c}$ and assume that

$$
D\left(\mathfrak{m}_{2, c}\right) \subseteq I=\left\langle y_{1}^{c}, y_{2}^{c}, \ldots, y_{c-1}^{c}\right\rangle=\gamma_{c}\left(\mathfrak{m}_{2, c}\right) .
$$

Moreover, by the fact that $D \in \operatorname{AID}\left(\mathfrak{m}_{2, c}\right)$ we must have that $D(x) \in\left[x, \mathfrak{m}_{2, c}\right]$ and hence

$$
D\left(x_{1}\right) \in\left\langle y_{1}^{c}, y_{2}^{c}, \ldots, y_{c-2}^{c}\right\rangle \text { and } D\left(x_{2}\right) \in\left\langle y_{2}^{c}, y_{3}^{c}, \ldots, y_{c-1}^{c}\right\rangle \text {. }
$$

So there are parameters $\alpha_{1}, \alpha_{2}, \ldots, \alpha_{c-2}, \beta_{2}, \beta_{3}, \ldots, \beta_{c-1} \in \mathbb{C}$ such that

$$
D\left(x_{1}\right)=\alpha_{1} y_{1}^{c}+\alpha_{2} y_{2}^{c}+\cdots+\alpha_{c-2} y_{c-2}^{c} \text { and } D\left(x_{2}\right)=\beta_{2} y_{2}^{c}+\beta_{3} y_{3}^{c}+\cdots+\beta_{c-1} y_{c-1}^{c} \text {. }
$$

By changing $D$ to $D-\operatorname{ad}_{\alpha_{1} y_{1}^{c-1}+\alpha_{2} y_{2}^{c-1}+\cdots+\alpha_{c-2} y_{c-2}^{c-1}}$, we may assume that all parameters $\alpha_{i}=0$ $(1 \leq i \leq c-2)$ and we are in the situation with

$$
D\left(x_{1}\right)=0 \text { and } D\left(x_{2}\right)=\beta_{2} y_{2}^{c}+\beta_{3} y_{3}^{c}+\cdots+\beta_{c-1} y_{c-1}^{c} .
$$


Now let $\lambda \in K$. Then on the one hand we have that

$$
D\left(\lambda x_{1}+x_{2}\right)=\lambda D\left(x_{1}\right)+D\left(x_{2}\right)=\beta_{2} y_{2}^{c}+\beta_{3} y_{3}^{c}+\cdots+\beta_{c-1} y_{c-1}^{c} .
$$

On the other hand, we also know that there exist an element $v_{\lambda} \in \mathfrak{m}_{2, c}$ with

$$
D\left(\lambda x_{1}+x_{2}\right)=\left[v_{\lambda}, \lambda x_{1}+x_{2}\right] .
$$

Let

$$
v_{\lambda}=a_{1} x_{1}+a_{2} x_{2}+\sum_{1 \leq n<m \leq c} a_{m, n} y_{n}^{m}
$$

then

$$
\left[v_{\lambda}, \lambda x_{1}+x_{2}\right]=\left(a_{2} \lambda-a_{1}\right) y_{1}^{2}+\sum_{1 \leq n<m \leq c-1} \lambda a_{m, n} y_{n}^{m+1}+\sum_{1 \leq n<m \leq c-1} a_{m, n} y_{n+1}^{m+1}
$$

Comparing the coefficients of the basis vectors $y_{i}^{c}$ of (5) with (6) we get the following system of equations:

$$
\left\{\begin{array}{l}
\lambda a_{c-1,1}=0 \\
\lambda a_{c-1,2}+a_{c-1,1}=\beta_{2} \\
\lambda a_{c-1,3}+a_{c-1,2}=\beta_{3} \\
\quad \vdots \\
\lambda a_{c-1, c-2}+a_{c-1, c-3}=\beta_{c-2} \\
a_{c-1, c-2}=\beta_{c-1}
\end{array}\right.
$$

This leads to

$$
\left\{\begin{array}{l}
\lambda a_{c-1,1}=0 \\
\lambda^{2} a_{c-1,2}+\lambda a_{c-1,1}=\lambda \beta_{2} \\
\lambda^{3} a_{c-1,3}+\lambda^{2} a_{c-1,2}=\lambda^{2} \beta_{3} \\
\quad \vdots \\
\lambda^{c-2} a_{c-1, c-2}+\lambda^{c-3} a_{c-1, c-3}=\lambda^{c-3} \beta_{c-2} \\
\lambda^{c-2} a_{c-1, c-2}=\lambda^{c-2} \beta_{c-1} .
\end{array}\right.
$$

By taking the alternating sum of all these equations, we find that

$$
\lambda \beta_{2}-\lambda^{2} \beta_{3}+\cdots+(-1)^{c-2} \lambda^{c-3} \beta_{c-2}+(-1)^{c-1} \lambda^{c-2} \beta_{c-1}=0 .
$$

Since the above equation has to hold for all possible $\lambda$ and $K$ is infinite, we must have that

$$
\beta_{2}=\beta_{3}=\cdots=\beta_{c-1}=0 .
$$

It follows that $D\left(x_{2}\right)=0$. Together with the fact that $D\left(x_{1}\right)=0$ this implies that $D=0$, which means that the original $D$ we started with was an inner derivation.

\section{Almost abelian Lie algebras and filiform nilpotent Lie algebras}

From now on we restrict ourselves to the case $K=\mathbb{C}$. Almost abelian Lie algebras have no unique definition in the literature. A common convention is that a Lie algebra $\mathfrak{g}$ is almost abelian if it contains a 1-codimensional abelian ideal. It is enough, however, to require that $\mathfrak{g}$ contains a 1-codimensional abelian subalgebra, see [5]. Here we consider almost inner derivations of complex almost abelian Lie algebras. We may write $\mathfrak{g}=\mathbb{C}^{n} \rtimes \mathbb{C}$ with $\mathbb{C}=\left\langle e_{n+1}\right\rangle$, and a basis 
$e_{1}, e_{2}, \ldots, e_{n}$ of $\mathbb{C}^{n}$, such that with respect to this basis, $\operatorname{ad}\left(e_{n+1}\right) \mathbb{C}^{n}$ is expressed in canonical Jordan form, i.e.,

$$
\operatorname{ad}\left(e_{n+1}\right)_{\mid \mathbb{C}^{n}}=\left(\begin{array}{ccccc}
B_{1} & 0 & 0 & \cdots & 0 \\
0 & B_{2} & 0 & \cdots & 0 \\
0 & 0 & B_{3} & \cdots & 0 \\
\vdots & \vdots & \vdots & \ddots & \vdots \\
0 & 0 & 0 & \cdots & B_{k}
\end{array}\right)
$$

where each $B_{i}$ is a block matrix of the form

$$
B_{i}=\left(\begin{array}{cccccc}
\lambda_{i} & 1 & 0 & \cdots & 0 & 0 \\
0 & \lambda_{i} & 1 & \cdots & 0 & 0 \\
0 & 0 & \lambda_{i} & \cdots & 0 & 0 \\
\vdots & \vdots & \vdots & \ddots & \vdots & \vdots \\
0 & 0 & 0 & \cdots & \lambda_{i} & 1 \\
0 & 0 & 0 & \cdots & 0 & \lambda_{i}
\end{array}\right)
$$

We can apply the lemmas on fixed vectors to prove the following result.

Proposition 7.1. Let $e_{1}, e_{2}, \ldots, e_{n}, e_{n+1}$ be the basis of $\mathfrak{g}=\mathbb{C}^{n} \rtimes \mathbb{C}$ as described above. Then for any almost inner derivation $D: \mathfrak{g} \rightarrow \mathfrak{g}$ determined by a map $\varphi_{D}$, any basis vector is fixed. It follows that $\operatorname{AID}(\mathfrak{g})=\operatorname{Inn}(\mathfrak{g})$.

Proof. Let $i \in\{1,2, \ldots, n\}$, then all basis vectors $e_{1}, e_{2}, \ldots, e_{n} \in C_{\mathfrak{g}}\left(e_{i}\right)$. Hence $e_{i}$ is fixed by Lemma 3.3. So it suffices to show that $e_{n+1}$ is fixed. Therefore, we need to show that for any $e_{j}, e_{k} \notin C_{\mathfrak{g}}\left(e_{n+1}\right)$ (with $1 \leq j<k \leq n$ ) we have that

$$
t_{n+1}\left(\varphi_{D}\left(e_{j}\right)\right)=t_{n+1}\left(\varphi_{D}\left(e_{k}\right)\right) .
$$

There are three different cases:

Case 1: $e_{j}$ and $e_{k}$ are basis vectors for different Jordan blocks. It follows that there exist $\lambda, \lambda^{\prime} \in \mathbb{C}$ such that

$$
\begin{aligned}
& {\left[e_{n+1}, e_{j}\right]=\lambda e_{j} \text { or } \lambda e_{j}+e_{j-1},} \\
& {\left[e_{n+1}, e_{k}\right]=\lambda^{\prime} e_{k} \text { or } \lambda^{\prime} e_{k}+e_{k-1} .}
\end{aligned}
$$

The two possibilities for each bracket are necessary for including the cases $\lambda=0$ or $\lambda^{\prime}=0$. In all of the situations above, we can use Lemma [3.7, with $l=j$ or $j-1$ and $m=k$ or $k-1$, to conclude that $t_{n+1}\left(\varphi_{D}\left(e_{j}\right)\right)=t_{n+1}\left(\varphi_{D}\left(e_{k}\right)\right)$.

Case 2: $e_{j}$ and $e_{k}$ are basis vectors for the same Jordan block and $k-j \geq 2$. In this case we have exactly the same conclusion as in the previous case.

Case 3: We have $k=j+1$ and $e_{j+1}$ and $e_{j}$ are basis vectors for the same Jordan block. In this case there is a $\lambda \in \mathbb{C}$ such that

$$
\begin{aligned}
{\left[e_{n+1}, e_{j+1}\right] } & =\lambda e_{j+1}+e_{j}, \\
{\left[e_{n+1}, e_{j}\right] } & =\lambda e_{j} \text { or } \lambda e_{j}+e_{j-1} .
\end{aligned}
$$

If $\lambda \neq 0$, then Lemma 3.8, with $l=j$ allows us to conclude that $t_{n+1}\left(\varphi_{D}\left(e_{j+1}\right)\right)=t_{n+1}\left(\varphi_{D}\left(e_{j}\right)\right)$. On the other hand, if $\lambda=0$, then we must have that $\left[e_{n+1}, e_{j+1}\right]=e_{j}$ and $\left[e_{n+1}, e_{j}\right]=e_{j-1}$, 
because otherwise $e_{j} \in C_{\mathfrak{g}}\left(e_{n+1}\right)$. In this case, we can again use Lemma 3.7, with $l=j-1$ and $m=j$, to conclude that $t_{n+1}\left(\varphi_{D}\left(e_{j+1}\right)\right)=t_{n+1}\left(\varphi_{D}\left(e_{j}\right)\right)$.

Denote by $\mathfrak{f}_{n}$ the standard graded filiform nilpotent Lie algebra of dimension $n$, defined by the Lie brackets $\left[e_{1}, e_{i}\right]=e_{i+1}$ for $i=2, \ldots, n-1$ in the basis $\left(e_{1}, \ldots, e_{n}\right)$. Clearly we have $\mathfrak{f}_{n} \cong \mathbb{C}^{n-1} \rtimes \mathbb{C}$ with $\mathbb{C}=\left\langle e_{1}\right\rangle$ over $\mathbb{C}$. Hence we obtain the following result as a corollary of Proposition 7.1 .

Proposition 7.2. The filiform nilpotent Lie algebra $\mathfrak{f}_{n}$ satisfies $\operatorname{AID}\left(\mathfrak{f}_{n}\right)=\operatorname{CAID}\left(\mathfrak{f}_{n}\right)=\operatorname{Inn}\left(\mathfrak{f}_{n}\right)$.

We already have seen in Example 2.7, that filiform nilpotent Lie algebras can have more interesting almost inner derivations than just inner ones. The algebra $\mathfrak{g}_{5,6}$ in this example is metabelian filiform. It turns out that this example generalizes to all metabelian filiform Lie algebras of dimension $n \geq 5$. It has been shown in [4] that every metabelian filiform Lie algebra $\mathfrak{g}$ of dimension $n \geq 3$ has an adapted basis $\left(e_{1}, \ldots, e_{n}\right)$ such that

$$
\begin{aligned}
& {\left[e_{1}, e_{i}\right]=e_{i+1}, 1 \leq i \leq n-1} \\
& {\left[e_{2}, e_{k}\right]=\alpha_{2,5} e_{2+k}+\cdots+\alpha_{2, n-k+3} e_{n}, 3 \leq k \leq n-2} \\
& {\left[e_{i}, e_{k}\right]=0, i, k \geq 3,}
\end{aligned}
$$

with structure constants $\left\{\alpha_{2, k} \mid 5 \leq k \leq n\right\}$. Clearly $\mathfrak{g} \cong \mathfrak{f}_{n}$ if and only if all structure constants are zero.

Lemma 7.3. Let $\mathfrak{g}$ be a complex metabelian filiform Lie algebra of dimension $n \geq 3$ and let $D \in \operatorname{AID}(\mathfrak{g})$. Then there exists a $v \in \mathfrak{g}$ and $a \lambda \in \mathbb{C}$ such that

$$
D-\operatorname{ad}_{v}=\lambda E_{n, 2} \text {. }
$$

Proof. We proceed by induction on the dimension $n$. If $n<5$, then $\mathfrak{g}$ is a standard filiform Lie algebra and all almost inner derivations are inner by Proposition 7.2 . So the result holds, with $\lambda=0$. So assume that $n \geq 5$ and that the lemma is valid for metabelian filiform Lie algebras of smaller dimensions. Let $D \in \operatorname{AID}(\mathfrak{g})$. Then $D$ induces an almost inner derivation $\bar{D}$ on $\mathfrak{g} /\left\langle e_{n}\right\rangle$. By induction, we may assume, after changing $D$ up to an inner derivation, that we have $\bar{D}=\mu E_{n-1,2}$ for some $\mu \in \mathbb{C}$. This implies that $D\left(e_{1}\right)=a e_{n}$ for some $a \in \mathbb{C}$. Now, replace $D$, with $D^{\prime}=D+\operatorname{ad}_{a e_{n-1}}$. Then we have

$$
\begin{aligned}
D^{\prime}\left(e_{1}\right) & =D\left(e_{1}\right)+\left[a e_{n-1}, e_{1}\right]=0, \\
D^{\prime}\left(e_{i}\right) & =D\left(e_{i}\right)+\left[a e_{n-1}, e_{i}\right]=D\left(e_{i}\right) \text { for } i \geq 2 .
\end{aligned}
$$

In particular, we have that

$$
D^{\prime}\left(e_{2}\right)=D\left(e_{2}\right)=\mu e_{n-1}+\lambda e_{n} \text { for some } \mu, \lambda \in \mathbb{C} .
$$

From this it follows that

$$
\begin{aligned}
& D^{\prime}\left(e_{3}\right)=D^{\prime}\left[e_{1}, e_{2}\right]=\left[D^{\prime}\left(e_{1}\right), e_{2}\right]+\left[e_{1}, D^{\prime}\left(e_{2}\right)\right]=\mu e_{n}, \\
& D^{\prime}\left(e_{4}\right)=D^{\prime}\left[e_{1}, e_{3}\right]=\left[D^{\prime}\left(e_{1}\right), e_{3}\right]+\left[e_{1}, D^{\prime}\left(e_{3}\right)\right]=0,
\end{aligned}
$$

and analogously $D^{\prime}\left(e_{i}\right)=0$ for $i \geq 5$. To finish the proof, we have to show that $\mu=0$. So assume that $\mu \neq 0$.

Since we have $D^{\prime}\left(e_{3}\right)=\mu e_{n}$ and $D^{\prime} \in \operatorname{AID}(\mathfrak{g})$, there must exist an element $\sum_{i=1}^{n} a_{i} e_{i} \in \mathfrak{g}$ with $\left[\sum_{i=1}^{n} a_{i} e_{i}, e_{3}\right]=\mu e_{n}$. This leads to the equation

$$
a_{1} e_{4}+a_{2}\left[e_{2}, e_{3}\right]=\mu e_{n},
$$


which expands to

$$
a_{1} e_{4}+a_{2}\left(\alpha_{2,5} e_{5}+\alpha_{2,6} e_{6}+\cdots+\alpha_{2, n} e_{n}\right)=\mu e_{n} .
$$

As we assume that $\mu \neq 0$, this implies

$$
\alpha_{2,5}=\alpha_{2,6}=\cdots=\alpha_{2, n-1}=0 .
$$

As a conclusion thus far, we have found that when $\mu \neq 0$, then the basis vectors $e_{i}$ satisfy

$$
\begin{aligned}
& {\left[e_{1}, e_{i}\right]=e_{i+1}, 2 \leq i \leq n-1} \\
& {\left[e_{2}, e_{3}\right]=\alpha_{2, n} e_{n}} \\
& {\left[e_{2}, e_{i}\right]=0,4 \leq i \leq n} \\
& {\left[e_{i}, e_{j}\right]=0, i, j \geq 3 .}
\end{aligned}
$$

There must also exist an element $\sum_{i=1}^{n} b_{i} e_{i} \in \mathfrak{g}$ with $D\left(e_{2}\right)=\left[\sum_{i=1}^{n} b_{i} e_{i}, e_{2}\right]$. This leads to the equation

$$
\mu e_{n-1}+\lambda e_{n}=b_{1} e_{3}-b_{3} \alpha_{2, n} e_{n}
$$

Since we are assuming that $\mu \neq 0$, this equation does not have a solution, which is a contradiction. Hence indeed $\mu=0$, and therefore $D^{\prime}=\lambda E_{2, n}$, which was to be shown.

The lemma now easily implies the following result.

Proposition 7.4. Let $\mathfrak{g}$ be a metabelian filiform Lie algebra of dimension $n \geq 5$, which is different from $\mathfrak{f}_{n}$. Then

$$
\operatorname{AID}(\mathfrak{g})=\operatorname{CAID}(\mathfrak{g})=\operatorname{Inn}(\mathfrak{g}) \oplus\left\langle E_{n, 2}\right\rangle .
$$

Proof. We only have to show that $D=E_{n, 2}$ is an almost inner derivation. If $x=\sum_{i=1}^{n} \beta_{i} e_{i}$ with $\beta_{1} \neq 0$, then $D(x)=\beta_{2} e_{n}=\left[x, \frac{\beta_{2}}{\beta_{1}} e_{n-1}\right]$. Otherwise $\beta_{1}=0$. Since $\mathfrak{g}$ is not the standard graded algebra $\mathfrak{f}_{n}$, there exists a minimal index $i$ with $5 \leq i \leq n$ such that $\alpha_{2, i} \neq 0$. Then, for $k=n-i+3 \geq 3$ we have $D(x)=\beta_{2} e_{n}=\left[x, \frac{1}{\alpha_{2, i}} e_{k}\right]$. Hence $D(x) \in[\mathfrak{g}, x]$ for all $x \in \mathfrak{g}$.

Remark 7.5. There are also filiform nilpotent Lie algebras $\mathfrak{g}$ with $\operatorname{dim}(\operatorname{AID}(\mathfrak{g}) / \operatorname{Inn}(\mathfrak{g})) \geq 2$ for $\operatorname{dim}(\mathfrak{g}) \geq 7$; of course with $d(\mathfrak{g}) \geq 3$. The following table shows the dimensions of the derivations spaces for all complex filiform nilpotent Lie algebras of dimension 7, with Magnin's notation [9]. For $\mathfrak{g}_{7,1.1\left(i_{\lambda}\right)}$ we have $\lambda \neq 0,1$. The two cases $\lambda=0$ and $\lambda=1$ are listed separately. The last column, when non zero, gives examples of almost inner derivations, which together with the inner derivations generate $\operatorname{AID}(\mathfrak{g})$.

\begin{tabular}{c|c|c|c|c|c|c|c} 
Magnin & $c(\mathfrak{g})$ & $d(\mathfrak{g})$ & $\operatorname{dim} \operatorname{Inn}(\mathfrak{g})$ & $\operatorname{dim} \operatorname{CAID}(\mathfrak{g})$ & $\operatorname{dim} \operatorname{AID}(\mathfrak{g})$ & $\operatorname{dim} \operatorname{Der}(\mathfrak{g})$ & $D$ \\
\hline $\mathfrak{g}_{7,0.1}$ & 6 & 3 & 6 & 7 & 8 & 10 & $E_{6,2}+E_{7,3}, E_{7,2}$ \\
\hline $\mathfrak{g}_{7,0.2}$ & 6 & 2 & 6 & 7 & 7 & 10 & $E_{7,2}$ \\
\hline $\mathfrak{g}_{7,0.3}$ & 6 & 2 & 6 & 7 & 7 & 11 & $E_{7,2}$ \\
\hline $\mathfrak{g}_{7,1.1\left(i_{\lambda}\right)}$ & 6 & 3 & 6 & 7 & 8 & 10 & $E_{6,2}+E_{7,3}, E_{7,2}$ \\
\hline $\mathfrak{g}_{7,1.1\left(i_{0}\right)}$ & 6 & 3 & 6 & 6 & 6 & 10 & 0 \\
\hline $\mathfrak{g}_{7,1.1\left(i_{1}\right)}$ & 6 & 2 & 6 & 7 & 7 & 11 & $E_{7,2}$ \\
\hline $\mathfrak{g}_{7,1.1(i i}$ & 6 & 3 & 6 & 7 & 7 & 11 & $E_{7,2}$ \\
\hline $\mathfrak{g}_{7,1.4}$ & 6 & 2 & 6 & 7 & 7 & 12 & $E_{7,2}$ \\
\hline $\mathfrak{g}_{7,1.6}$ & 6 & 2 & 6 & 7 & 7 & 12 & $E_{7,2}$ \\
\hline $\mathfrak{g}_{7,2.3}$ & 6 & 2 & 6 & 6 & 6 & 13 & 0 \\
\hline
\end{tabular}




\section{LoW-Dimensional LiE ALGEBRAS}

Complex Lie algebras of dimension $n \leq 4$ do not have non-inner almost inner derivations. This is different in dimension 5. In order to determine the space of almost inner derivations we will not use a full classification of all 5-dimensional Lie algebras, but rather a description of the moduli space given in [6]. Here the authors give a natural stratification by orbifolds, in terms of 24 families of Lie algebras, with up to 4 parameters. This is much better than a full classification for us, because the determination of almost inner derivations is much more efficient for the stratification, the list of the full classification being much too long. We already know from the table after Example 2.7 that every complex nilpotent Lie algebra of dimension 5 having a non-inner almost inner derivation is isomorphic to $\mathfrak{g}_{5,3}$ or $\mathfrak{g}_{5,6}$.

The most interesting family of solvable, non-nilpotent Lie algebras in this context is the family $d_{12}(p: q: r)$ with $p=0$ from [6].

Definition 8.1. The family of complex 5-dimensional Lie algebras $A(q, r)=d_{12}(0: q: r)$ is defined by the Lie brackets

$$
\begin{aligned}
& {\left[e_{1}, e_{5}\right]=e_{2},} \\
& {\left[e_{2}, e_{5}\right]=(q+r) e_{2},} \\
& {\left[e_{3}, e_{4}\right]=e_{2}} \\
& {\left[e_{3}, e_{5}\right]=e_{1}+q e_{3},} \\
& {\left[e_{4}, e_{5}\right]=e_{3}+r e_{4} .}
\end{aligned}
$$

It is straightforward to compute the almost inner derivations of this family.

Lemma 8.2. We have

$$
\begin{aligned}
& \operatorname{dim} \operatorname{Der}(A(q, r))= \begin{cases}7, & \text { if }(q, r) \neq(0,0) \\
8, & \text { otherwise }\end{cases} \\
& \operatorname{dim} \operatorname{Inn}(A(q, r))=4, \quad \text { for all } q, r \in \mathbb{C} \\
& \operatorname{dim} \operatorname{AID}(A(q, r))= \begin{cases}4, & \text { if } q r \neq 0, q+r \neq 0 \\
5, & \text { otherwise }\end{cases}
\end{aligned}
$$

We can determine the Lie algebras $A(q, r)$ with $\operatorname{dim} \operatorname{AID}(A(q, r))=5$ up to isomorphism.

Lemma 8.3. Every Lie algebra $A(q, r)$ satisfying $q r=0$ or $q+r=0$ is either isomorphic to $A(1,0)$, to $A(1,-1)$ or to $A(0,0) \cong \mathfrak{g}_{5,6}$.

Proof. Note that $A(q, r) \cong A(r, q)$, see [6]. It is easy to see that $A(0,0)$ is filiform nilpotent and isomorphic to $\mathfrak{g}_{5,6}$. So we may assume that $(q, r) \neq(0,0)$. Suppose first that $q r=0$. Then we may assume $q \neq 0$ and $r=0$, and there is an Lie algebra isomorphism $\varphi: A(q, 0) \rightarrow A(1,0)$ given by

$$
\left(\begin{array}{ccccc}
q^{2} & 0 & 0 & 0 & 0 \\
1-q^{2} & q & 0 & 0 & 0 \\
0 & 0 & q & 0 & 0 \\
0 & 0 & 0 & 1 & \frac{1-q^{2}}{q} \\
0 & 0 & 0 & 0 & q
\end{array}\right)
$$


Secondly, let $q+r=0$ and $q \neq 0$. Then there is an Lie algebra isomorphism $\varphi: A(q,-q) \rightarrow$ $A(1,-1)$ given by

$$
\left(\begin{array}{ccccc}
1 & 0 & \frac{q^{2}-1}{q} & \frac{q^{2}-1}{q^{2}} & 0 \\
0 & q & \frac{q^{2}-1}{q} & 0 & 0 \\
0 & 0 & q & 0 & 0 \\
0 & 0 & 0 & 1 & 0 \\
0 & 0 & 0 & 0 & q
\end{array}\right) .
$$

Finally, $A(q, r)$ is unimodular if and only if $q+r=0$. Hence $A(1,-1)$ is unimodular, but $A(1,0)$ is not. So they cannot be isomorphic. Both $A(1,-1)$ and $A(1,0)$ are solvable and non-nilpotent, whereas $A(0,0)$ is nilpotent.

Proposition 8.4. Every complex Lie algebra of dimension 5 having a non-inner almost inner derivation is isomorphic to one of the following Lie algebras:

$$
\mathfrak{g}_{5,3}, \mathfrak{g}_{5,6}, A(1,0), A(1,-1) .
$$

Proof. We use Table 3 of [6] listing the 24 families of Lie algebras. For each family, or type, we compute the spaces $\operatorname{Der}(\mathfrak{g}), \operatorname{AID}(\mathfrak{g})$ and $\operatorname{Inn}(\mathfrak{g})$ for all possible parameters. The types $d_{1}, d_{2}, d_{3}, d_{4}, d_{7}, d_{8}, d_{10}, d_{11}, d_{16}, d_{17}, d_{18}, d_{19}, d_{24}$ have no parameters, so that the computation is easy. Also, the nilpotent algebras are easy, because they correspond to choosing all parameters equal to zero. Moreover we do know the result already for nilpotent algebras. Note that there is an error in the Lie brackets of $d_{2}$ in table 3 of [6], where $\psi_{1}^{12}$ has to be removed; and also in the definition on page 429. The hardest cases are the ones with 3 or 4 parameters, namely the families $d_{5}(p: q: r), d_{12}(p: q: r), d_{20}(p: q: r: s)$ and $d_{21}(p: q: r)$. A long, but straightforward computation shows that, for non-nilpotent algebras, the only family with noninner almost inner derivations is $d_{12}(p: q: r)$, where we need $p=0$. More precisely we see that only for the algebras $A(q, r)=d_{12}(0: q: r)$ with $q=0$, or $r=0$ or $q+r=0$ this is the case. We obtain (see Lemma 8.2)

$$
\operatorname{AID}(A(q, r))= \begin{cases}\operatorname{Inn}(A(q, r)) \oplus\left\langle E_{2,4}\right\rangle & \text { for } q=0, r \neq 0 \\ \operatorname{Inn}(A(q, r)) \oplus\left\langle E_{2,4}+q E_{3,5}\right\rangle & \text { for } r=0, q \neq 0 \\ \operatorname{Inn}(A(q, r)) \oplus\left\langle r^{2} E_{2,1}-E_{2,4}+r E_{3,5}+r^{2} E_{4,5}\right\rangle & \text { for } q+r=0\end{cases}
$$

Remark 8.5. The Lie algebra $A(1,-1)$ arises in a different context, namely in the classification of Lie algebras admitting a Sasakian structure, see [1. A Sasakian structure on a Riemannian manifold is the analogue in odd dimensions of a Kähler structure. Indeed, a Riemannian manifold $M$ of odd dimension admits a compatible Sasakian structure if and only if the Riemannian cone $M \times \mathbb{R}^{+}$is Kähler. Left-invariant Sasakian structures on Lie groups can be classified by Sasakian structures on its Lie algebras. In the classification of 5-dimensional Sasakin Lie algebras in Theorem 10 of [1], the Sasakian Lie algebra $\mathfrak{g}_{3}$ is isomorphic to $A(1,-1)$ over the complex numbers.

Remark 8.6. In dimension 6 we have computed the almost inner derivations only for nilpotent Lie algebras, using the classification given in [9]. The result is given in the following table: 


\begin{tabular}{c|c|c|c|c|c|c|c} 
Magnin & $c(\mathfrak{g})$ & $d(\mathfrak{g})$ & $\operatorname{dim} \operatorname{Inn}(\mathfrak{g})$ & $\operatorname{dim} \operatorname{CAID}(\mathfrak{g})$ & $\operatorname{dim} \operatorname{AID}(\mathfrak{g})$ & $\operatorname{dim} \operatorname{Der}(\mathfrak{g})$ & $D$ \\
\hline $\mathfrak{g}_{6,20}$ & 5 & 3 & 5 & 5 & 6 & 8 & $E_{5,2}$ \\
\hline $\mathfrak{g}_{6,18}$ & 5 & 3 & 5 & 5 & 5 & 9 & 0 \\
\hline $\mathfrak{g}_{6,19}$ & 5 & 2 & 5 & 6 & 6 & 9 & $E_{6,2}$ \\
\hline $\mathfrak{g}_{6,17}$ & 5 & 2 & 5 & 6 & 6 & 10 & $E_{6,2}$ \\
\hline $\mathfrak{g}_{6,15}$ & 4 & 2 & 5 & 5 & 5 & 10 & 0 \\
\hline $\mathfrak{g}_{6,13}$ & 4 & 2 & 5 & 6 & 6 & 10 & $E_{6,3}$ \\
\hline $\mathfrak{g}_{6,16}$ & 5 & 2 & 5 & 5 & 5 & 11 & 0 \\
\hline $\mathfrak{g}_{6,14}$ & 4 & 2 & 4 & 4 & 4 & 11 & 0 \\
\hline $\mathfrak{g}_{6,9}$ & 3 & 2 & 5 & 5 & 5 & 11 & 0 \\
\hline $\mathfrak{g}_{6,12}$ & 4 & 2 & 5 & 5 & 5 & 11 & 0 \\
\hline $\mathfrak{g}_{5,6} \oplus \mathbb{C}$ & 4 & 2 & 4 & 5 & 5 & 12 & $E_{5,2}$ \\
\hline $\mathfrak{g}_{6,5}$ & 3 & 2 & 4 & 4 & 4 & 12 & 0 \\
\hline $\mathfrak{g}_{6,10}$ & 3 & 2 & 5 & 5 & 5 & 12 & 0 \\
\hline $\mathfrak{g}_{6,11}$ & 4 & 2 & 5 & 6 & 6 & 12 & $E_{6,3}$ \\
\hline $\mathfrak{g}_{5,5} \oplus \mathbb{C}$ & 4 & 2 & 4 & 4 & 4 & 13 & 0 \\
\hline $\mathfrak{g}_{6,8}$ & 3 & 2 & 4 & 6 & 6 & 13 & $E_{5,3}, E_{6,2}$ \\
\hline $\mathfrak{g}_{6,4}$ & 3 & 2 & 4 & 4 & 4 & 13 & 0 \\
\hline $\mathfrak{g}_{6,7}$ & 3 & 2 & 4 & 6 & 6 & 14 & $E_{6,2}, E_{6,3}$ \\
\hline $\mathfrak{g}_{6,2}$ & 3 & 2 & 5 & 5 & 5 & 14 & 0 \\
\hline $\mathfrak{g}_{6,6}$ & 3 & 2 & 4 & 4 & 4 & 15 & 0 \\
\hline $\mathfrak{g}_{5,4} \oplus \mathbb{C}$ & 3 & 2 & 3 & 3 & 3 & 15 & 0 \\
\hline $\mathfrak{g}_{5,3} \oplus \mathbb{C}$ & 3 & 2 & 4 & 5 & 5 & 15 & $E_{5,3}$ \\
\hline $\mathfrak{n}_{3} \oplus \mathfrak{n}_{3}$ & 2 & 2 & 4 & 4 & 4 & 16 & 0 \\
\hline $\mathfrak{n}_{4} \oplus \mathbb{C}$ & 3 & 2 & 3 & 3 & 3 & 17 & 0 \\
\hline $\mathfrak{g}_{6,1}$ & 2 & 2 & 4 & 6 & 6 & 17 & $E_{6,3}, E_{6,4}$ \\
\hline $\mathfrak{g}_{6,3}$ & 2 & 2 & 3 & 3 & 3 & 18 & 0 \\
\hline $\mathfrak{g}_{5,2} \oplus \mathbb{C}$ & 2 & 2 & 3 & 3 & 3 & 19 & 0 \\
\hline $\mathfrak{g}_{5,1} \oplus \mathbb{C}$ & 2 & 2 & 4 & 4 & 4 & 21 & 0 \\
\hline $\mathfrak{n}_{3} \oplus \mathbb{C} 3$ & 2 & 2 & 2 & 2 & 2 & 24 & 0 \\
\hline $\mathbb{C}_{6}$ & 1 & 1 & 0 & 0 & 0 & 36 & 0 \\
\hline
\end{tabular}

\section{Triangular Lie Algebras}

In this section we consider the Lie algebra $\mathfrak{t}_{n}(K)$, resp. $\mathfrak{n}_{n}(K)$, of all upper-triangular, resp. strictly upper-triangular, $n \times n$ matrices over a general field $K$ again.

Let $e_{i, j}$ denote the $n \times n$ matrix with 0's everywhere, except a 1 on the $(i, j)$-th spot. Recall that

$$
\left[e_{i, j}, e_{k, l}\right]=\delta_{j, k} e_{i, l}-\delta_{l, i} e_{k, j} .
$$

Proposition 9.1. For any $n \geq 2$ we have that

$$
\operatorname{AID}\left(\mathfrak{n}_{n}(K)\right)=\operatorname{Inn}\left(\mathfrak{n}_{n}(K)\right) .
$$

Proof. The Lie algebra $\mathfrak{n}_{n}(K)$ has a basis consisting of the matrices $e_{i, j}$ where $1 \leq i<j \leq n$. From (7) it follows that

$$
\left[\mathfrak{n}_{n}(K), e_{i, j}\right]=\left\langle e_{i, j+1}, e_{i, j+2}, \ldots, e_{i, n}, e_{1, j}, e_{2, j}, \ldots, e_{i-1, j}\right\rangle .
$$


We proceed by induction on $n$. For $n=2$ the proposition is trivially true since $\mathfrak{n}_{2}(K) \cong K$ is abelian. So we assume that $n>2$ and that the result holds for smaller values of $n$.

Let $D \in \operatorname{AID}\left(\mathfrak{n}_{n}(K)\right)$. Note that $I=\left\langle e_{1,2}, e_{1,3}, \ldots, e_{1, n}\right\rangle$ is an ideal of $\mathfrak{n}_{n}(K)$. This implies that $D(I) \subseteq I$. It follows that $D$ induces a derivation $\bar{D}$ of $\mathfrak{n}_{n}(K) / I \cong \mathfrak{n}_{n-1}(K)$. Of course $\bar{D} \in$ $\operatorname{AID}\left(\mathfrak{n}_{n-1}(K)\right)$ and by induction, we can conclude that $\bar{D}$ is an inner derivation. Let $x \in \mathfrak{n}_{n}(K)$ be an element such that $\bar{D}=\operatorname{ad}(\bar{x})$ where $\bar{x}$ denotes the projection of $x$ in $\mathfrak{n}_{n-1}(K) \cong \mathfrak{n}_{n}(K) / I$. By replacing $D$ by $D-\operatorname{ad}(x)$ we may assume that $D$ is an almost inner derivation of $\mathfrak{n}_{n}(K)$ with $D\left(\mathfrak{n}_{n}(K)\right) \subseteq I$. It follows that there exist elements $\beta_{3}, \beta_{4}, \ldots, \beta_{n} \in K$ such that

$$
D\left(e_{2,3}\right)=\beta_{3} e_{1,3}, D\left(e_{3,4}\right)=\beta_{4} e_{1,4}, D\left(e_{4,5}\right)=\beta_{5} e_{1,5}, \ldots, D\left(e_{n-1, n}\right)=\beta_{n} e_{1, n} .
$$

Let $a=\beta_{3} e_{1,2}+\beta_{4} e_{1,3}+\cdots+\beta_{n} e_{1, n-1}$, then for all $i$ with $2 \leq i \leq n-1$ we have that

$$
\begin{aligned}
\operatorname{ad}(a)\left(e_{i, i+1}\right) & =\left[\beta_{3} e_{1,2}+\beta_{4} e_{1,3}+\cdots+\beta_{n} e_{1, n-1}, e_{i, i+1}\right] \\
& =\left[\beta_{i+1} e_{1, i}, e_{i, i+1}\right] \\
& =\beta_{i+1} e_{1, i+1} \\
& =D\left(e_{i, i+1}\right) .
\end{aligned}
$$

So, by replacing $D$ with $D-\operatorname{ad}(a)$, we may assume that

$$
D\left(e_{2,3}\right)=D\left(e_{3,4}\right)=\cdots=D\left(e_{n-1, n}\right)=0 .
$$

Note that $\operatorname{ad}(a)(I) \subseteq I$, so that also after modifying $D$, we still have that $D\left(\mathfrak{n}_{n}(K)\right) \subseteq I$.

There also exist $\alpha_{3}, \alpha_{4}, \ldots, \alpha_{n} \in K$ with

$$
D\left(e_{1,2}\right)=\alpha_{3} e_{1,3}+\alpha_{4} e_{1,4}+\cdots+\alpha_{n} e_{1, n} .
$$

For $3 \leq i<n$ we have $\left[e_{1,2}, e_{i, i+1}\right]=0$, so that

$$
\begin{aligned}
0 & =D\left[e_{1,2}, e_{i, i+1}\right] \\
& =\left[D\left(e_{1,2}\right), e_{i, i+1}\right]+\left[e_{1,2}, D\left(e_{i, i+1}\right)\right] \\
& =\left[\alpha_{3} e_{1,3}+\alpha_{4} e_{1,4}+\cdots+\alpha_{n} e_{1, n}, e_{i, i+1}\right]+0 \\
& =\alpha_{i} e_{1, i+1} .
\end{aligned}
$$

It follows that $\alpha_{i}=0$ for all $n>i \geq 3$, so that

$$
D\left(e_{1,2}\right)=\alpha_{n} e_{1, n}=\operatorname{ad}\left(-\alpha_{n} e_{2, n}\right)\left(e_{1,2}\right) .
$$

Note that for $i \geq 2$ we have $\operatorname{ad}\left(-\alpha_{n} e_{2, n}\right)\left(e_{i, i+1}\right)=0$. So by finally replacing $D$ with $D+$ $\operatorname{ad}\left(\alpha_{n} e_{2, n}\right)$ we find that

$$
D\left(e_{i, i+1}\right)=0, \forall 1 \leq i<n .
$$

But this implies that $D=0$, so that the original $D$ is in $\operatorname{Inn}\left(\mathfrak{n}_{n}(K)\right)$, which was to be shown.

By exactly the same technique one can also prove the following result:

Proposition 9.2. For any $n \geq 2$ we have

$$
\operatorname{AID}\left(\mathfrak{t}_{n}(K)\right)=\operatorname{Inn}\left(\mathfrak{t}_{n}(K)\right)
$$




\section{Nilpotent Lie algebras with arbitrary large $\operatorname{AID}(\mathfrak{g}) / \operatorname{Inn}(\mathfrak{g})$}

In the previous sections we had many negative results concerning the existence of non-inner almost inner derivations. We want to show now that it is also possible to construct infinite families of Lie algebras $\mathfrak{g}$ having a $\operatorname{space} \operatorname{AID}(\mathfrak{g}) / \operatorname{Inn}(\mathfrak{g})$ of arbitrarily large dimension $n$, for any given $n \in \mathbb{N}$.

Consider the following family of 2-step nilpotent Lie algebras $\mathfrak{g}_{n}$ over a general field $K$ of dimension $4 n+2$, with basis

$$
t_{1}, t_{2}, x_{1, i}, x_{2, i}, y_{1, i}, y_{2, i}(1 \leq i \leq n)
$$

and non-zero Lie brackets

$$
\left[t_{1}, x_{1, i}\right]=y_{1, i},\left[t_{1}, x_{2, i}\right]=y_{2, i},\left[t_{2}, x_{2, i}\right]=y_{1, i}(1 \leq i \leq n) .
$$

So we have $\mathfrak{g}_{n}=K^{4 n} \rtimes K^{2}$, where $K^{4 n}$ is the subspace spanned by the $x_{p, i}$ 's and the $y_{p, i}$ 's and $K^{2}$ is spanned by $t_{1}$ and $t_{2}$.

Proposition 10.1. For every $n \geq 2$ we have

$$
\operatorname{dim}\left(\operatorname{AID}\left(\mathfrak{g}_{n}\right) / \operatorname{Inn}\left(\mathfrak{g}_{n}\right)\right)=n .
$$

Proof. Any element $x$ of $\mathfrak{g}_{n}$ can be written uniquely in the form

$$
x=\alpha_{1} t_{1}+\alpha_{2} t_{2}+v
$$

where $v \in K^{4 n}=\left\langle x_{p, i}, y_{p, i}, 1 \leq p \leq 2,1 \leq i \leq n\right\rangle$. Using this notation we define for any $i=1,2, \ldots, n$ a map

$$
\varphi_{D_{i}}: \mathfrak{g}_{n} \rightarrow \mathfrak{g}_{n}: x=\alpha_{1} t_{1}+\alpha_{2} t_{2}+v \mapsto\left\{\begin{array}{lll}
0 & \text { if } & \alpha_{1}=0 \\
-\frac{\alpha_{2}}{\alpha_{1}} x_{1, i}+x_{2, i} & \text { if } & \alpha_{1} \neq 0
\end{array}\right.
$$

Now let

$$
D_{i}: \mathfrak{g}_{n} \rightarrow \mathfrak{g}_{n}: x \mapsto D_{i}(x):=\left[x, \varphi_{D_{i}}(x)\right]
$$

For $\alpha_{1} \neq 0$ we have that

$$
\begin{aligned}
D_{i}\left(\alpha_{1} t_{1}+\alpha_{2} t_{2}+v\right) & =\left[\alpha_{1} t_{1}+\alpha_{2} t_{2}+v,-\frac{\alpha_{2}}{\alpha_{1}} x_{1, i}+x_{2, i}\right] \\
& =-\alpha_{2} y_{1, i}+\alpha_{1} y_{2, i}+\alpha_{2} y_{1, i} \\
& =\alpha_{1} y_{2, i}
\end{aligned}
$$

Also for $\alpha_{1}=0$, we have that $D_{i}\left(\alpha_{1} t_{1}+\alpha_{2} t_{2}+v\right)=0=\alpha_{1} y_{2, i}$. Hence $D_{i}: \mathfrak{g}_{n} \rightarrow \mathfrak{g}_{n}$ is a linear map, having its image in the center of $\mathfrak{g}_{n}$, and so $D_{i}$ is a derivation. By construction, $D_{i} \in \operatorname{AID}\left(\mathfrak{g}_{n}\right)$. We claim that the set of all $D_{i}+\operatorname{Inn}\left(\mathfrak{g}_{n}\right)(1 \leq i \leq n)$ forms a basis of $\operatorname{AID}\left(\mathfrak{g}_{n}\right) / \operatorname{Inn}\left(\mathfrak{g}_{n}\right)$.

We will first show that this is a linearly independent set. Assume that $\sum_{i=1}^{n} \beta_{i} D_{i} \in \operatorname{Inn}\left(\mathfrak{g}_{n}\right)$, then

$$
\sum_{i=1}^{n} \beta_{i} D_{i}=\operatorname{ad}\left(\alpha_{1} t_{1}+\alpha_{2} t_{2}+v\right)
$$


for some $\alpha_{1}, \alpha_{2} \in K, v \in K^{4 n}$. As $\sum_{i=1}^{n} \beta_{i} D_{i}\left(x_{1,1}\right)=0$, it follows that $0=\left[\alpha_{1} t_{1}+\alpha_{2} t_{2}+v, x_{1,1}\right]=$ $\alpha_{1} y_{1,1}$, so that $\alpha_{1}=0$. Analogously, the fact that $\sum_{i=1}^{n} \beta_{i} D_{i}\left(x_{2,1}\right)=0$ now leads to $\alpha_{2}=0$, so $\sum_{i=1}^{n} \beta_{i} D_{i}=\operatorname{ad}(v)$ for some $v \in K^{4 n}$. But then

$$
\begin{aligned}
\sum_{i=1}^{n} \beta_{i} y_{2, i} & =\sum_{i=1}^{n} \beta_{i} D_{i}\left(t_{1}\right)=\left[v, t_{1}\right], \\
0 & =\sum_{i=1}^{n} \beta_{i} D_{i}\left(t_{2}\right)=\left[v, t_{2}\right] .
\end{aligned}
$$

The second equation above shows that $v$ has no components in the $x_{2, i}$ 's and thus is $\left[v, t_{1}\right]=0$. Using this in the first equation above leads to $\beta_{1}=\beta_{2}=\cdots=\beta_{n}=0$.

Next we have to verify that the set is generating. Let $D \in \operatorname{AID}\left(\mathfrak{g}_{n}\right)$. We have to show that

$$
D=\sum_{i=1}^{n} \beta_{i} D_{i}+\operatorname{ad}(x)
$$

for some $\beta_{1}, \beta_{2}, \ldots, \beta_{n} \in K$ and $x \in \mathfrak{g}_{n}$. Let $D$ be determined by a map $\varphi_{D}$. Many of the basis vectors turn out to be fixed:

1. As any vector $y_{p, q}$ belongs to the center of $\mathfrak{g}_{n}$, all of these basis vectors are fixed.

2. Also any vector $x_{1, i}$ is fixed, since its centralizer is of codimension 1 in $\mathfrak{g}_{n}$, see Lemma 3.3.

3. To see that $t_{2}$ is fixed, note that the basis vectors not belonging to $C_{\mathfrak{g}_{n}}\left(t_{2}\right)$ are the vectors $x_{2, i}$. When we apply Lemma 3.7 with $e_{i}=t_{2}, e_{j}=x_{2, i}, e_{k}=x_{2, j}, e_{l}=y_{1, i}$ and $e_{m}=y_{1, j}$ we can deduce that

$$
t_{t_{2}}\left(\varphi_{D}\left(x_{2, i}\right)\right)=t_{t_{2}}\left(\varphi_{D}\left(x_{2, j}\right)\right)
$$

from which is follows that $t_{2}$ is fixed.

4. To see that $t_{1}$ is fixed, we start with applying Lemma 3.7 with $e_{i}=t_{1}, e_{j}=x_{1, i}, e_{k}=x_{1, j}$, $e_{l}=y_{1, i}$ and $e_{m}=y_{1, j}$. This gives us that

$$
t_{t_{1}}\left(\varphi_{D}\left(x_{1, i}\right)\right)=t_{t_{1}}\left(\varphi_{D}\left(x_{1, j}\right)\right) \text { for all } 1 \leq i, j \leq n .
$$

Now applying Lemma 3.7 for $i \neq j$ with with $e_{i}=t_{1}, e_{j}=x_{1, i}, e_{k}=x_{2, j}, e_{l}=y_{1, i}$ and $e_{m}=y_{2, j}$ we find that

$$
t_{t_{1}}\left(\varphi_{D}\left(x_{1, i}\right)\right)=t_{t_{1}}\left(\varphi_{D}\left(x_{2, j}\right)\right) \text { for all } 1 \leq i, j \leq n \text { with } i \neq j .
$$

Together with the above (and knowing that $n \geq 2$ ) we can conclude that

$$
t_{t_{1}}\left(\varphi_{D}\left(x_{1, i}\right)\right)=t_{t_{1}}\left(\varphi_{D}\left(x_{1, j}\right)\right)=t_{t_{1}}\left(\varphi_{D}\left(x_{2, k}\right)\right) \text { for all } 1 \leq i, j, k \leq n,
$$

showing that $t_{1}$ is fixed.

The only basisvectors which are not fixed are the vectors $x_{2, i}$. By applying Lemma 3.5 for every fixed basis vector, we may now assume that, after changing $D$ up to an inner derivation, we have for each basis vector $x$ that $\varphi_{D}(x)=\sum_{i=1}^{n} \beta_{i}(x) x_{2, i}$ for some $\beta_{i}(x) \in K$. By changing $D$ to $D+\operatorname{ad}\left(\varphi_{D}\left(t_{2}\right)\right)$, we may suppose that $\varphi_{D}\left(t_{2}\right)=0$, and so $D\left(t_{2}\right)=\left[t_{2}, \varphi_{D}\left(t_{2}\right)\right]=0$. Let $x$ be one of the basis vectors $x_{p, i}$ or $y_{p, i}$, then also

$$
D(x)=\left[x, \varphi_{D}(x)\right]=\left[x, \sum_{i=1}^{n} \beta_{i}(x) x_{2, i}\right]=0 .
$$


Finally, we have that

$$
D\left(t_{1}\right)=\left[t_{1}, \varphi_{D}\left(t_{1}\right)\right]=\left[t_{1}, \sum_{i=1}^{n} \beta_{i}\left(t_{1}\right) x_{2, i}\right]=\sum_{i=1}^{n} \beta_{i}\left(t_{1}\right) y_{2, i} .
$$

As a conclusion, we find that, after changing $D$ up to an inner derivation, we obtain

$$
D=\sum_{i=1}^{n} \beta_{i}\left(t_{1}\right) D_{i}
$$

Remark 10.2. For $n=1$ the basis vector $t_{1}$ is not fixed. Then the algebra $\mathfrak{g}_{1}$ of the above family is isomorphic to $\mathfrak{g}_{6,1}$ of Remark 8.6, which is also the algebra of Example $(i)$ of [7], page 245. For this algebra we know that $\operatorname{dim}\left(\operatorname{AID}\left(\mathfrak{g}_{1}\right) / \operatorname{Inn}\left(\mathfrak{g}_{1}\right)\right)=2$.

\section{ACKNowledgements}

The first author was supported in part by the Austrian Science Fund (FWF), grant P28079 and grant I3248. The second and third author are supported by long term structural funding - Methusalem grant of the Flemish Government.

\section{REFERENCES}

[1] A. Andrada, A. Fino, L. Vezzoni: A class of Sasakian 5-manifolds. Transformation groups, Vol. 14 (2009), no. 3, 493-512.

[2] S. Ayupov, K. Kudaybergenov: Local derivations on finite-dimensional Lie algebras. Linear Algebra Appl. 493 (2016), 381-398.

[3] Y.A. Bahturin: Identical relations in Lie algebras. VNU Science Press b.v. (Utercht), 1987.

[4] F. Bratzlavsky: Classification des algébres de Lie de dimension $n$, de classe $n-1$, dont l'idéal dérivé est commutativ, Bull. Cl. Sci. Bruxelles 60 (1974), 858-865.

[5] D. Burde, M. Ceballos: Abelian ideals of maximal dimension for solvable Lie algebras. Journal of Lie Theory 22 (2012), no. 3, 741-756.

[6] A. Fialowski, M. Penkava: The moduli space of complex 5-dimensional Lie algebras. J. Algebra 458 (2016), $422-444$.

[7] C. S. Gordon, E. N. Wilson: Isospectral deformations of compact solvmanifolds. J. Differential Geom. 19 (1984), no. 1, 214-256.

[8] M. Larsen; On the conjugacy of element-conjugate homomorphisms. Israel J. Math. 88(1994), no. 1-3, $253-277$.

[9] L. Magnin: Adjoint and Trivial Cohomology Tables for Indecomposable Nilpotent Lie Algebras of Dimension $\leq 7$ over $\mathbb{C}$. Online e-book, 2d Corrected Edition (2007), 810+vi pages.

[10] J. Milnor: Eigenvalues of the Laplace operator on certain manifolds. Proc. Nat. Acad. Sci. U.S.A. 51 (1964), $542 \mathrm{ff}$.

Fakultät für Mathematik, Universität Wien, Oskar-Morgenstern-Platz 1, 1090 Wien, AusTRIA

E-mail address: dietrich.burde@univie.ac.at

Katholieke Universiteit Leuven Kulak, E. Sabbelaan 53 bus 7657, 8500 Kortrijk, Belgium

E-mail address: karel.dekimpe@kuleuven-kulak.be

Katholieke Universiteit Leuven Kulak, E. Sabbelaan 53 bus 7657, 8500 Kortrijk, Belgium

E-mail address: bert.verbeke@kuleuven-kulak. be 